Pacific Journal of Mathematic 


\section{REGULAR BOUNDARY PROBLEMS FOR A FIVE-TERM RECURRENCE RELATION}

\section{E. BILLIGHEIMER}

We consider in this paper boundary problems for the fiveterm scalar recurrence relation

$$
\begin{gathered}
d_{n} y_{n+2}+c_{n} y_{n+1}+\left(b_{n}-\lambda a_{n}\right) y_{n}+c_{n-1} y_{n-1}+d_{n-2} y_{n-2}=0 \\
(0 \leqq n \leqq m)
\end{gathered}
$$

where the coefficients $a_{n}, b_{n}, c_{n}, d_{n}$ are real, $a_{n}, d_{n}>0$ and $\lambda$ is a complex parameter, with boundary conditions of the typical form

$$
y_{-2}=y_{-1}=0
$$

and

$$
y_{m+1}+k\left(c_{m} y_{m}+d_{m-1} y_{m-1}\right)=0, y_{m+2}+h y_{m}=0
$$

for some integer $m \geqq 0$, and real numbers $h, k$.

We derive oscillation properties, orthogonality relations and associated eigenvector expansion theorems for solutions of (1.1), (1.2), (1.3), and then discuss the solution of boundary problems for the corresponding inhomogeneous recurrence relation in terms of a Green's function.

Atkinson [1] has discussed the connection between two and three term scalar and matrix recurrence relations and Sturm-Liouville differential equations and first order systems of differential equations.

On this basis the five-term recurrence relation here discussed appears as the analogue of a fourth order differential equation or first order system of dimension four.

The self-adjoint second order differential equation for which the fundamental limit-point, limit-circle distinction for the singular boundary problem first given by Weyl [12] plays an important part is discussed in detail for example in the work of Coddington and Levinson [5]. The analogous three-term recurrence relations were studied by Stone [11] in the setting of Hilbert space theory.

The extension of the theory to the case of the general even order differential equation was given by Kodaira [9] Glazman [8] and Everitt [6], [7] who studied also in particular the fourth order case. A fundamental study of the oscillation theory of the fourth order differential equation was made by Leighton and Nehari [10] and Barrett [2], [3].

We discuss in this paper regular boundary problems for the fiveterm recurrence relation (1.1). In a subsequent paper (Billigheimer 
[4]) we consider singular boundary problems for the recurrence relation (1.1) with (1.2) as $m \rightarrow \infty$.

2. The boundary problem. We discuss firstly fundamental properties of the boundary problem for the recurrence relations (1.1), (1.2), (1.3).

We define the fundamental solution vectors $p_{n}(\lambda), q_{n}(\lambda), s_{n}(\lambda), r_{n}(\lambda)$ of (1.1), polynomials in $\lambda$ with real coefficients, by

$$
\begin{aligned}
& p_{-2}(\lambda)=p_{-1}(\lambda)=0, p_{0}(\lambda)=1, p_{1}(\lambda)=0 ; \\
& q_{-2}(\lambda)=q_{-1}(\lambda)=0, q_{0}(\lambda)=0, q_{1}(\lambda)=1 ; \\
& s_{-2}(\lambda)=0, s_{-1}(\lambda)=1, s_{0}(\lambda)=s_{1}(\lambda)=0 ; \\
& r_{-2}(\lambda)=1, r_{-1}(\lambda)=0, r_{0}(\lambda)=r_{1}(\lambda)=0 .
\end{aligned}
$$

The vector $y_{n}(\lambda)=\alpha p_{n}(\lambda)+\beta q_{n}(\lambda)$, where $\alpha, \beta$ are constants, satisfies the recurrence relations (1.1) and the initial conditions (1.2) and is the most general such solution. $y_{n}(\lambda)$ will satisfy the boundary conditions (1.3) if

$$
\begin{aligned}
\alpha\left\{p_{m+1}(\lambda)\right. & \left.+k\left[c_{m} p_{m}(\lambda)+d_{m-1} p_{m-1}(\lambda)\right]\right\} \\
& +\beta\left\{q_{m+1}(\lambda)+k\left[c_{m} q_{m}(\lambda)+d_{m-1} q_{m-1}(\lambda)\right]\right\}=0 \\
\alpha\left\{p_{m+2}(\lambda)\right. & \left.+h p_{m}(\lambda)\right\}+\beta\left\{q_{m+2}(\lambda)+h q_{m}(\lambda)\right\}=0 .
\end{aligned}
$$

We now define for two vectors $u=\left\{u_{n}\right\}_{-2}^{m+2}, v=\left\{v_{n}\right\}_{-2}^{m+2}$ the form $|u v|_{n}(n \geqq-1)$ by

$$
|u v|_{n}=\left|\begin{array}{ll}
u_{n+1}+k\left(c_{n} u_{n}+d_{n-1} u_{n-1}\right) & v_{n+1}+k\left(c_{n} v_{n}+d_{n-1} v_{n-1}\right) \\
u_{n+2}+h u_{n} & v_{n+2}+h v_{n}
\end{array}\right|(n \geqq-1) .
$$

Then for $\alpha, \beta$ not both zero in the above equations we require that the polynomial in $\lambda, \rho_{m}(\lambda)(m \geqq 0)$, defined by

$$
\rho_{m}(\lambda)=|p(\lambda) q(\lambda)|_{m} \quad(m \geqq 0)
$$

should be zero. We shall refer to the polynomial $\rho_{m}(\lambda)$ as the characteristic polynomial for the boundary problem (1.1), (1.2), (1.3).

$\rho_{m}(\lambda)$ is a polynomial of true degree $m+1$ in $\lambda$ with real coefficients. Hence there are always $m+1$ roots, counted according to multiplicity, of the equation $\rho_{m}(\lambda)=0$. We call those roots eigenvalues of the boundary problem (1.1), (1.2), (1.3) and the associated solution vectors of the recurrence relations eigenvectors.

We show later that the eigenvalues $\lambda_{r}$ are all real and that for repeated roots $\lambda_{r}$ of $\rho_{m}(\lambda)=0$ we have two linearly independent eigenvectors. We call single roots of $\rho_{m}(\lambda)=0$ simple eigenvalues of the boundary problem (1.1), (1.2), (1.3) and double roots double 
eigenvalues. We shall see that triple eigenvalues are not possible.

We now define two vector polynomials in $\lambda$ in terms of which eigenvectors of the boundary problem (1.1), (1.2), (1.3) can be expressed.

We form for general $\lambda$, not necessarily an eigenvalue, the solution vector $y_{n}^{(m)}(\lambda)$ of the recurrence relations (1.1) satisfying (1.2)

$$
y_{n}^{(m)}(\lambda)=\left\{q_{m+2}(\lambda)+h q_{m}(\lambda)\right\} p_{n}(\lambda)-\left\{p_{m+2}(\lambda)+h p_{m}(\lambda)\right\} q_{n}(\lambda)
$$

for $n \geqq-2$.

$y_{n}^{(m)}(\lambda)$ is a polynomial of true degree $\frac{1}{2}(m+n+1)$ if precisely one of $m, n$ is odd and of apparent degree $\frac{1}{2}(m+n)$ if both $m, n$ are odd or even. We see that

$$
y_{m+2}^{(m)}(\lambda)+h y_{m}^{(m)}(\lambda)=0
$$

for all $\lambda$, and hence in particular also

$$
y_{m+2}^{(m)^{\prime}}(\lambda)+h y_{m}^{(m)^{\prime}}(\lambda)=0 \text {. }
$$

Also

$$
y_{m+1}^{(m)}(\lambda)+k\left[c_{m} y_{m}^{(m)}(\lambda)+d_{m-1} y_{m-1}^{(m)}(\lambda)\right]=\rho_{m}(\lambda)
$$

and is hence zero for eigenvalues $\lambda_{r}$ of the boundary problem (1.1), (1.2), (1.3).

Hence if at least one of $p_{m+2}\left(\lambda_{r}\right)+h p_{m}\left(\lambda_{r}\right), q_{m+2}\left(\lambda_{r}\right)+h q_{m}\left(\lambda_{r}\right)$ is not equal to zero, the vector $y_{n}^{(m)}\left(\lambda_{r}\right)(-2 \leqq n \leqq m+2)$ furnishes a nontrivial real eigenvector for the boundary problem (1.1), (1.2), (1.3).

We also form the vector polynomial

$$
\begin{aligned}
z_{n}^{(m)}(\lambda)= & \left\{q_{m+1}(\lambda)+k\left[c_{m} q_{m}(\lambda)+d_{m-1} q_{m-1}(\lambda)\right]\right\} p_{n}(\lambda) \\
& -\left\{p_{m+1}(\lambda)+k\left[c_{m} p_{m}(\lambda)+d_{m-1} p_{m-1}(\lambda)\right]\right\} q_{n}(\lambda)
\end{aligned}
$$

for $n \geqq-2$.

$\boldsymbol{Z}_{n}^{(m)}(\lambda)$ is a polynomial of true degree $\frac{1}{2}(m+n)$ if both $m, n$ are odd or even and of apparent degree $\frac{1}{2}(m+n-1)$ if precisely one of $m, n$ is odd

$$
z_{m+1}^{(m)}(\lambda)+k\left[c_{m} z_{m}^{(m)}(\lambda)+d_{m-1} z_{m-1}^{(m)}(\lambda)\right]=0
$$

for all $\lambda$, and hence in particular

$$
\boldsymbol{z}_{m+1}^{(m)^{\prime}}(\lambda)+k\left[c_{m} z_{m}^{(m)^{\prime}}(\lambda)+d_{m-1} z_{m-1}^{(m)^{\prime}}(\lambda)\right]=0 .
$$

Also

$$
\boldsymbol{z}_{m+2}^{(m)}(\lambda)+h \boldsymbol{z}_{m}^{(m)}(\lambda)=-\rho_{m}(\lambda) .
$$

Hence if, for $\lambda_{r}$ an eigenvalue of (1.1), (1.2), (1.3) for which $\rho_{m}\left(\lambda_{r}\right)=0$, at least one of $p_{m+1}\left(\lambda_{r}\right)+k\left[c_{m} p_{m}\left(\lambda_{r}\right)+d_{m-1} p_{m-1}\left(\lambda_{r}\right)\right]$, 
$q_{m+2}\left(\lambda_{r}\right)+h\left[c_{m} q_{m}\left(\lambda_{r}\right)+d_{m-1} q_{m-1}\left(\lambda_{r}\right)\right]$ is not equal to zero, the vector $z_{n}^{(m)}\left(\lambda_{r}\right)(-2 \leqq n \leqq m+2)$ furnishes a nontrivial real eigenvector for (1.1), (1.2), (1.3).

At values of $\lambda$ other than roots of $\rho_{m}(\lambda)=0, y_{n}^{(m)}(\lambda)$ and $z_{n}^{(m)}(\lambda)$ are two linearly independent solution vectors of the recurrence relations (1.1) satisfying (1.2), provided that they are not trivially zero vectors. At an eigenvalue $\lambda_{r}$ of (1.1), (1.2), (1.3) they are proportional unless one or both are trivially zero. At a simple eigenvalue $\lambda_{r}$ at least one of the vectors $y_{n}^{(m)}\left(\lambda_{r}\right), z_{n}^{(m)}\left(\lambda_{r}\right)$ provides a nontrivial eigenvector as we see from (2.2). At a repeated eigenvalue $\lambda_{r}$ both $y_{n}^{(m)}\left(\lambda_{r}\right)$ and $z_{n}^{(m)}\left(\lambda_{r}\right)$ are the trivially zero vector as we shall see from later results. In this case $p_{n}\left(\lambda_{r}\right), q_{n}\left(\lambda_{r}\right)$ are linearly independent real eigenvectors. We shall see that at most a doubly repeated eigenvalue can occur.

3. Fundamental identities. We now obtain for the polynomials previously defined certain relations of the type of the ChristoffelDarboux relations for orthogonal polynomials analogous to the Lagrange identities for differential equations.

We define for two sequences $u=\left\{u_{n}\right\}, v=\left\{v_{n}\right\}(n \geqq-2)$ the form $[u v]_{n}(n \geqq-1)$ by

$$
[u v]_{n}=d_{n}\left|\begin{array}{ll}
u_{n+2} & \bar{v}_{n+2} \\
u_{n} & \bar{v}_{n}
\end{array}\right|+c_{n}\left|\begin{array}{ll}
u_{n+1} & \bar{v}_{n+1} \\
u_{n} & \bar{v}_{n}
\end{array}\right|+d_{n-1}\left|\begin{array}{ll}
u_{n+1} & \bar{v}_{n+1} \\
u_{n-1} & \bar{v}_{n-1}
\end{array}\right| \text {. }
$$

Then we have

Lemma 3.1. For $u(\lambda), v(\mu)$ any two solution vectors of the recurrence relations (1.1) with parameter $\lambda, \mu$ respectively, we have

$$
(\lambda-\mu) \sum_{r=0}^{n} a_{r} u_{r}(\lambda) v_{r}(\mu)=[u(\lambda) \bar{v}(\mu)]_{n}-[u(\lambda) \bar{v}(\mu)]_{-1} \quad(n \geqq 0) .
$$

Proof. We have

$$
\begin{aligned}
& d_{r} u_{r+2}(\lambda)+c_{r} u_{r+1}(\lambda)+\left(b_{r}-\lambda a_{r}\right) u_{r}(\lambda)+c_{r-1} u_{r-1}(\lambda)+d_{r-2} u_{r-2}(\lambda)=0 \\
& d_{r} v_{r+2}(\mu)+c_{r} v_{r+1}(\mu)+\left(b_{r}-\mu a_{r}\right) v_{r}(\mu)+c_{r-1} v_{r-1}(\mu)+d_{r-2} v_{r-2}(\mu)=0 .
\end{aligned}
$$

Multiplying the first equation by $v_{r}(\mu)$ and the second by $u_{r}(\lambda)$, subtracting and summing over $r$ from 0 to $n$ we obtain (3.2).

Similarly we obtain

LEMMA 3.2. For $u(\lambda)$ any solution vector of (1.1) with $\operatorname{Im} \lambda \neq 0$ we have

$$
\sum_{r=0}^{n} a_{r} u_{r}(\lambda) \bar{u}_{r}(\lambda)=(2 i \operatorname{Im} \lambda)^{-1}\left\{[u(\lambda) u(\lambda)]_{n}-[u(\lambda) u(\lambda)]_{-1}\right\} \quad(n \geqq 0)
$$


Proof. Consider equation (1.1) and its complex conjugate for $u(\lambda)$ as in the proof of Lemma 3.1.

\section{From Lemma 3.1 we deduce}

LEMma 3.3. For $u(\lambda), v(\lambda)$ two differentiable solution vectors of (1.1) we have

$$
\begin{aligned}
\sum_{r=0}^{n} a_{r} u_{r}(\lambda) v_{r}(\lambda) & =\left[u^{\prime}(\lambda) \bar{v}(\lambda)\right]_{n}-\left[u^{\prime}(\lambda) \bar{v}(\lambda)\right]_{-1} \\
& =-\left[u(\lambda) \bar{v}^{\prime}(\lambda)\right]_{n}+\left[u(\lambda) \bar{v}^{\prime}(\lambda)\right]_{-1} \quad(n \geqq 0) .
\end{aligned}
$$

Proof. Use l'Hospital's rule or differentiate (3.2) with respect to $\lambda$ and let $\mu \rightarrow \lambda$.

From Lemma 3.1 we also obtain setting $\mu=\lambda$

Lemma 3.4. For $u(\lambda), v(\lambda)$ any two solution vectors of (1.1) we have

$$
[u(\lambda) \bar{v}(\lambda)]_{n}-[u(\lambda) \bar{v}(\lambda)]_{-1}=0 \quad(n \geqq-1) .
$$

Hence we can define for two solution vectors $u(\lambda), v(\lambda)$ of (1.1) for fixed $\lambda$ the function independent of $n$

$$
[u \bar{v}]=[u(\lambda) \bar{v}(\lambda)]=[u(\lambda) \bar{v}(\lambda)]_{n} \quad(n \geqq-1) .
$$

We see from (3.1) that $[u \bar{u}]=0$.

In particular for the solution vectors $p(\lambda), q(\lambda), r(\lambda)$ and $s(\lambda)$ defined in (2.1) we have

$$
[p \bar{q}]=0,[p \bar{r}]=d_{-2},[p \bar{s}]=c_{-1},[q \bar{r}]=0,[q \bar{s}]=d_{-1},[r \bar{s}]=0 .
$$

We now use the above relations to deduce the following lemma:

Lemma 3.5. Let $u(\lambda), v(\lambda)$ be two differentiable solutions of the recurrence relations (1.1) with $a_{n}, d_{n}>0$ which are real for real $\lambda$ and which satisfy the initial conditions $u_{-2}(\lambda)=0, u_{-1}(\lambda)=0$, and $v_{-2}(\lambda)=0, v_{-1}(\lambda)=0$ for all $\lambda$.

Let $\lambda$ be an eigenvalue of the boundary problem (1.1), (1.2), (1.3) for some $m \geqq 0$ with $u(\lambda), v(\lambda)$ both eigenvectors. Suppose $\lambda$ is real. Then we have the inequality

$$
\begin{aligned}
d_{m} d_{m-1}\left|\begin{array}{ll}
u_{m+1}^{\prime}+k\left(c_{m} u_{m}^{\prime}+d_{m-1} u_{m-1}^{\prime}\right) & v_{m+1}^{\prime}+k\left(c_{m} v_{m}^{\prime}+d_{m-1} v_{m-1}^{\prime}\right) \\
u_{m+2}^{\prime}+h u_{m}^{\prime} & v_{m+2}^{\prime}+h v_{m}^{\prime}
\end{array}\right| \\
\quad \times\left|\begin{array}{ll}
u_{m-1} & v_{m-1} \\
u_{m} & v_{m}
\end{array}\right| \geqq 0
\end{aligned}
$$


where equality holds if and only if the solutions $u(\lambda), v(\lambda)$ are proportional.

Proof. We use the algebraic identity

$$
\left(\sum_{r} a_{r} u_{r}^{2}\right)\left(\sum_{s} a_{s} v_{s}^{2}\right)-\left(\sum_{r} a_{r} u_{r} v_{r}\right)^{2}=\sum_{r, s} a_{r} a_{s}\left(u_{r} v_{s}-u_{s} v_{r}\right)^{2} \geqq 0 .
$$

Using the identity (3.4) with the relations (1.1), (1.2), (1.3) satisfied by $u(\lambda), v(\lambda)$ we have

$$
\begin{aligned}
& \left(\sum_{r=0}^{m} a_{r} u_{r}^{2}\right)\left(\sum_{s=0}^{m} a_{s} v_{s}^{2}\right)-\left(\sum_{r=0}^{m} a_{r} u_{r} v_{r}\right)^{2} \\
& =\left[u^{\prime} \bar{u}\right]_{m}\left[v^{\prime} \bar{v}\right]_{m}+\left[u^{\prime} \bar{v}\right]_{m}\left[u \bar{v}^{\prime}\right]_{m} \\
& =d_{m}\left|\begin{array}{ll}
u_{m+2}^{\prime} & v_{m+2}^{\prime} \\
u_{m}^{\prime} & v_{m}^{\prime}
\end{array}\right|\left|\begin{array}{ll}
d_{m} u_{m+2}+c_{m} u_{m+1} & d_{m} v_{m+2}+c_{m} v_{m+1} \\
u_{m} & v_{m}
\end{array}\right| \\
& +d_{m-1}\left|\begin{array}{ll}
u_{m+1}^{\prime} & v_{m+1}^{\prime} \\
u_{m}^{\prime} & v_{m}^{\prime}
\end{array}\right|\left|\begin{array}{ll}
d_{m} u_{m+2}+c_{m} u_{m+1} & d_{m} v_{m+2}+c_{m} v_{m+1} \\
u_{m-1} & v_{m-1}
\end{array}\right| \\
& +c_{m}\left|\begin{array}{ll}
u_{m+1}^{\prime} & v_{m+1}^{\prime} \\
u_{m}^{\prime} & v_{m}^{\prime}
\end{array}\right|\left|\begin{array}{ll}
d_{m} u_{m+2}+c_{m} u_{m+1} & d_{m} v_{m+2}+c_{m} v_{m+1} \\
u_{m} & v_{m}
\end{array}\right| \\
& +d_{m} d_{m-1}\left|\begin{array}{ll}
u_{m+2}^{\prime} & v_{m+2}^{\prime} \\
u_{m+1}^{\prime} & v_{m+1}^{\prime}
\end{array}\right|\left|\begin{array}{ll}
u_{m} & v_{m} \\
u_{m-1} & v_{m-1}
\end{array}\right| \\
& +d_{m} d_{m-1}\left|\begin{array}{ll}
u_{m+2}^{\prime} & v_{m+2}^{\prime} \\
u_{m-1}^{\prime} & v_{m-1}^{\prime}
\end{array}\right|\left|\begin{array}{ll}
u_{m+1} & v_{m+1} \\
u_{m} & v_{m}
\end{array}\right| \\
& +d_{m-1}\left|\begin{array}{ll}
u_{m+1}^{\prime} & v_{m+1}^{\prime} \\
u_{m-1}^{\prime} & v_{m-1}^{\prime}
\end{array}\right| \begin{array}{ll}
u_{m+1} & v_{m+1} \\
c_{m} u_{m}+d_{m-1} u_{m-1} & c_{m} v_{m}+d_{m-1} v_{m-1}
\end{array} \mid \\
& +d_{m} d_{m-1}\left|\begin{array}{ll}
u_{m}^{\prime} & v_{m}^{\prime} \\
u_{m-1}^{\prime} & v_{m-1}^{\prime}
\end{array}\right|\left|\begin{array}{ll}
u_{m+2} & v_{m+2} \\
u_{m+1} & v_{m+1}
\end{array}\right| \\
& =d_{m} d_{m-1}\left|\begin{array}{ll}
u_{m+1}^{\prime}+k\left(c_{m} u_{m}^{\prime}+d_{m-1} u_{m-1}^{\prime}\right) & v_{m+1}^{\prime}+k\left(c_{m} v_{m}^{\prime}+d_{m-1} v_{m-1}^{\prime}\right) \\
u_{m+2}^{\prime}+h u_{m}^{\prime} & v_{m+2}^{\prime}+h v_{m}^{\prime}
\end{array}\right| \\
& \times\left|\begin{array}{ll}
u_{m-1} & v_{m-1} \\
u_{m} & v_{m}
\end{array}\right|=\sum_{r, s=0}^{m} a_{r} a_{s}\left(u_{r} v_{s}-u_{s} v_{r}\right)^{2} \geqq 0
\end{aligned}
$$

for real solutions $u$, $v$, with equality if and only if the solutions $u, v$ are proportional.

We note that by Theorem 4.1 of the following section the eigenvalues of the boundary problem (1.1), (1.2), (1.3) are real.

The lemma yields the corollary:

COROLlaRY. If the fundamental solution vectors $p_{n}(\lambda), q_{n}(\lambda)$ of (1.1), (1.2) satisfy for some real $\lambda$ also the boundary conditions (1.3) with $m \geqq 0$ and the same $h, k$, then we have the inequality 


$$
\begin{aligned}
d_{m} d_{m-1}\left|\begin{array}{ll}
p_{m+1}^{\prime}+k\left(c_{m} p_{m}^{\prime}+d_{m-1} p_{m-1}^{\prime}\right) & q_{m+1}^{\prime}+k\left(c_{m} q_{m}^{\prime}+d_{m-1} q_{m-1}^{\prime}\right) \\
p_{m+2}^{\prime}+h p_{m}^{\prime} & q_{m+2}^{\prime}+h q_{m}^{\prime}
\end{array}\right| \\
\quad \times\left|\begin{array}{ll}
p_{m-1} & q_{m-1} \\
p_{m} & q_{m}
\end{array}\right|>0
\end{aligned}
$$

Proof. The vectors $p(\lambda), q(\lambda)$ are not proportional for any $\lambda$ since $p_{0}(\lambda)=1, p_{1}(\lambda)=0, q_{0}(\lambda)=0, q_{1}(\lambda)=1$. Hence taking $u=p(\lambda), v=q(\lambda)$ in Lemma 3.5 we obtain the statement of the corollary (3.9) with strict inequality.

4. Properties of characteristic roots. We now use the fundamental relations obtained in the preceding sections to derive some fundamental theorems concerning the eigenvalues and eigenvectors of the boundary problem (1.1), (1.2), (1.3).

Theorem 4.1. The eigenvalues of the boundary problem (1.1), (1.2), (1.3) are all real.

Proof. Assume $\lambda_{s}$ is a root of the characteristic equation

$$
\rho_{m}(\lambda)=0
$$

where $\rho_{m}(\lambda)$ is defined in (2.2).

Then $\lambda_{s}$ is an eigenvalue of the boundary problem (1.1), (1.2), (1.3). Thus we have a nontrivial eigenvector $u\left(\lambda_{s}\right)$ of the boundary problem.

Assume $\lambda_{s}$ is nonreal. From Lemma 3.2 with $\lambda=\lambda_{s}$ and with $n=m$ we obtain that the right-hand side of (3.2) is 0 and hence the left side is also 0 . Since $a_{r}>0$ we have $u_{r}\left(\lambda_{s}\right)=0(r=0,1, \cdots, m)$ and hence $u\left(\lambda_{s}\right)$ is the trivially zero vector. This is a contradiction to our assumptions.

Hence we must have $\operatorname{Im}\left(\lambda_{s}\right)$ is zero and $\lambda_{s}$ is real.

THEOREM 4.2. If $\lambda_{s}$ is a repeated eigenvalue of the boundary problem (1.1), (1.2), (1.3) then $p_{n}\left(\lambda_{s}\right), q_{n}\left(\lambda_{s}\right)$ are linearly independent eigenvectors of the boundary problem (1.1), (1.2), (1.3), and conversely.

Proof. Assume

$$
\rho_{m}\left(\lambda_{s}\right)=\rho_{m}^{\prime}\left(\lambda_{s}\right)=0 .
$$

Assume $p_{m+2}\left(\lambda_{s}\right)+h p_{m}\left(\lambda_{s}\right)$ and $q_{m+2}\left(\lambda_{s}\right)+h q_{m}\left(\lambda_{s}\right)$ are not both 0 . Then $y_{n}^{(m)}\left(\lambda_{s}\right)$ as defined in (2.3) is a nontrivial eigenvector of the problem (1.1), (1.2), (1.3). $y_{n}^{(m)}\left(\lambda_{s}\right)$ satisfies (2.4), (2.5) and (2.6) and we hence have in this case using (4.2) that 


$$
\begin{gathered}
y_{m+2}^{(m)}\left(\lambda_{s}\right)+h y_{m}^{(m)}\left(\lambda_{s}\right)=0, y_{m+2}^{(m) \prime}\left(\lambda_{s}\right)+h y_{m}^{(m) \prime}\left(\lambda_{s}\right)=0 \\
y_{m+1}^{(m)}\left(\lambda_{s}\right)+k\left[c_{m} y_{m}^{(m)}\left(\lambda_{s}\right)+d_{m-1} y_{m-1}^{(m)}\left(\lambda_{s}\right)\right]=0, y_{m+1}^{(m) \prime}\left(\lambda_{s}\right)+k\left[c_{m} y_{m}^{(m) \prime}\left(\lambda_{s}\right)\right. \\
\left.+d_{m-1} y_{m-1}^{(m) \prime}\left(\lambda_{s}\right)\right]=0 .
\end{gathered}
$$

If we now set $\lambda=\lambda_{s}, u_{r}(\lambda)=v_{r}(\lambda)=y_{r}^{(m)}(\lambda), n=m$ in (3.4) we obtain a contradiction since the right-hand side becomes 0 while the left-hand side is positive. Hence we deduce that

$$
p_{m+2}\left(\lambda_{s}\right)+h p_{m}\left(\lambda_{s}\right)=0 \text { and } q_{m+2}\left(\lambda_{s}\right)+h q_{m}\left(\lambda_{s}\right)=0 \text {. }
$$

Now assume $p_{m+1}\left(\lambda_{s}\right)+k\left[c_{m} p_{m}\left(\lambda_{s}\right)+d_{m-1} p_{m-1}\left(\lambda_{s}\right)\right]$ and $q_{m+1}\left(\lambda_{s}\right)+$ $k\left[c_{m} q_{m}\left(\lambda_{s}\right)+d_{m-1} q_{m-1}\left(\lambda_{s}\right)\right]$ are not both zero. Then $z_{n}^{(m)}\left(\lambda_{s}\right)$ as defined in (2.7) is a nontrivial eigenvector of the problem (1.1), (1.2), (1.3). $z_{n}^{(m)}\left(\lambda_{s}\right)$ satisfies (2.8), (2.9) and (2.10) and we have hence in this case using (4.2) that

$$
\begin{gathered}
\boldsymbol{z}_{m+1}^{(m)}\left(\lambda_{s}\right)+k\left[c_{m} \boldsymbol{z}_{m}^{(m)}\left(\lambda_{s}\right)+d_{m-1} \boldsymbol{z}_{m-1}^{(m)}\left(\lambda_{s}\right)\right]=0, \\
\boldsymbol{z}_{m+1}^{(m) \prime}\left(\lambda_{s}\right)+k\left[c_{m} \boldsymbol{z}_{m}^{(m)^{\prime}}\left(\lambda_{s}\right)+d_{m-1} z_{m-1}^{(m)^{\prime}}\left(\lambda_{s}\right)\right]=0, \\
\boldsymbol{z}_{m+2}^{(m)}\left(\lambda_{s}\right)+h \boldsymbol{z}_{m}^{(m)}\left(\lambda_{s}\right)=0, \boldsymbol{z}_{m+2}^{(m)^{\prime}}\left(\lambda_{s}\right)+h \boldsymbol{z}_{m}^{(m)^{\prime}}\left(\lambda_{s}\right)=0 .
\end{gathered}
$$

If we now set $\lambda=\lambda_{s}, v_{r}(\lambda)=v_{r}(\lambda)=z_{r}^{(m)}\left(\lambda_{s}\right), n=m$ in (3.4) we obtain again a contradiction. Hence we deduce that

(4.4) and

$$
\begin{aligned}
& p_{m+1}\left(\lambda_{s}\right)+k\left[c_{m} p_{m}\left(\lambda_{s}\right)+d_{m-1} p_{m-1}\left(\lambda_{s}\right)\right]=0 \\
& q_{m+1}\left(\lambda_{s}\right)+k\left[c_{m} q_{m}\left(\lambda_{s}\right)+d_{m-1} q_{m-1}\left(\lambda_{s}\right)\right]=0 .
\end{aligned}
$$

Hence from (4.3), (4.4) we deduce that $p_{n}\left(\lambda_{s}\right)$ and $q_{n}\left(\lambda_{s}\right)$ are both eigenvectors of (1.1), (1.2), (1.3) and are of course linearly independent.

The converse is obvious as from (2.2) the equations (4.3), (4.4) quarantee $\rho_{m}\left(\lambda_{s}\right)=0=\rho_{m}^{\prime}\left(\lambda_{s}\right)$.

THeOREM 4.3. The boundary problem (1.1), (1.2), (1.3) can have at most a double eigenvalue.

Proof. At a repeated root $\lambda_{s}$ of the characteristic equation (4.1) with $\rho_{m}\left(\lambda_{s}\right)=0=\rho_{m}^{\prime}\left(\lambda_{s}\right)$ we have from (2.2)

$$
\begin{aligned}
& \rho_{m}^{\prime \prime}\left(\lambda_{s}\right)= \\
& 2\left|\begin{array}{lc}
p_{m+1}^{\prime}\left(\lambda_{s}\right)+k\left[c_{m} p_{m}^{\prime}\left(\lambda_{s}\right)+d_{m-1} p_{m-1}^{\prime}\left(\lambda_{s}\right)\right] q_{m+1}^{\prime}\left(\lambda_{s}\right)+k\left[c_{m} q_{m}^{\prime}\left(\lambda_{s}\right)+d_{m-1} q_{m-1}^{\prime}\left(\lambda_{s}\right)\right] \\
p_{m+2}^{\prime}\left(\lambda_{s}\right)+h p_{m}^{\prime}\left(\lambda_{s}\right) & q_{m+2}^{\prime}\left(\lambda_{s}\right)+h q_{m}^{\prime}\left(\lambda_{s}\right)
\end{array}\right| .
\end{aligned}
$$

By the corollary to Lemma 3.5 and since $\lambda_{s}$ is real by Theorem 4.1 we have that $\rho_{m}^{\prime \prime}\left(\lambda_{s}\right) \neq 0$ and hence we can have at most a doubly 
repeated root of (4.1). Alternatively from (1.1) it follows that there can be at most two linearly independent eigenvectors.

5. Oscillation and separation theorems. We use the relations of $\S 3$ and the theorems of $\S 4$ to obtain some results concerning the oscillation and separation properties for solutions of the boundary problem (1.1), (1.2), and (1.3) in the special case $h=0=k$. We first derive the following lemmas:

Lemma 5.1. It is not possible for real $\lambda$ that $p_{m+1}(\lambda)=q_{m+1}(\lambda)=$ $p_{m+2}(\lambda)=q_{m+2}(\lambda)=0$ and also $p_{m}(\lambda)=q_{m}(\lambda)=0$ for $m \geqq 0$.

Proof. We use the corollary to Lemma 3.5. We note that this lemma is a particular case of the general result of Lemma 5.4 which is valid for all $\lambda$. Hence the statement of Lemma 5.1 is also true for nonreal $\lambda$.

LEMMA 5.2. It is not possible that $y_{m}(\lambda)=y_{m+1}(\lambda)=y_{m+2}(\lambda)=$ $y_{m+3}(\lambda)=0(m \geqq-2)$, for any nontrivial solution $y_{n}(\lambda)$ of the recurrence relations (1.1).

Proof. We see directly from (1.1) that a solution with four consecutive components equal to 0 must have all components equal to 0 .

LEMMA 5.3. It is not possible that $y_{m}(\lambda)=y_{m+1}(\lambda)=y_{m+2}(\lambda)=$ $y_{m+1}^{\prime}(\lambda)=0(m \geqq 0)$ if $y_{n}(\lambda)$ is a nontrivial and real differentiable solution of the recurrence relations (1.1) and (1.2) for real $\lambda$.

Proof. We use formula (3.4) and Lemma 5.2 to demonstrate the lemma. Under the assumptions the right-hand side of (3.4) for $n=m$ is 0 . Hence the left-hand side is 0 and $y_{0}=y_{1}=\cdots=y_{m}=0$ with $m \geqq 0$. For $m \geqq 1$ we use $y_{m+1}=y_{m+2}=0$ and for $m=0$ also from (1.2) $y_{-2}=y_{-1}=0$ to deduce from Lemma 5.2 that in each case $y(\lambda)$ is the trivially zero vector.

LEMMA 5.4. If two solution vectors of (1.1), (1.2) have three successive components in common then they are proportional.

Proof. From (1.1) we see that a solution with three successive zero components is determined uniquely by the value of an adjacent component.

We now have the following theorems also for the special case of 
the problem (1.1), (1.2), (1.3) with $h=k=0$ :

THEOREM 5.1. If $\lambda_{s}$ is a repeated root of $\rho_{m}(\lambda)$ for $m \geqq 1$ then $\lambda_{s}$ is a simple root of $\rho_{m-1}(\lambda)$ and of $\rho_{m+1}(\lambda)$.

Proof. At a repeated root $\lambda_{s}$ of $\rho_{m}(\lambda)$ we have from Theorem 4.2 that $p_{m+1}\left(\lambda_{s}\right)=q_{m+1}\left(\lambda_{s}\right)=p_{m+2}\left(\lambda_{s}\right)=q_{m+2}\left(\lambda_{s}\right)=0$. Then we see from (2.2) that also $\rho_{m-1}(\lambda)$ and $\rho_{m+1}(\lambda)$ are 0 at $\lambda=\lambda_{s}$.

By the corollary to Lemma 3.5 we have that

$$
\left|\begin{array}{ll}
p_{m-1}\left(\lambda_{s}\right) & q_{m-1}\left(\lambda_{s}\right) \\
p_{m}\left(\lambda_{s}\right) & q_{m}\left(\lambda_{s}\right)
\end{array}\right| \neq 0
$$

Using (1.1) we see that this implies that

$$
\left|\begin{array}{ll}
p_{m+3}\left(\lambda_{s}\right) & q_{m+3}\left(\lambda_{s}\right) \\
p_{m}\left(\lambda_{s}\right) & q_{m}\left(\lambda_{s}\right)
\end{array}\right| \neq 0
$$

and it is impossible that $p_{m}\left(\lambda_{s}\right)=q_{m}\left(\lambda_{s}\right)=0$ or $p_{m+3}\left(\lambda_{s}\right)=q_{m+3}\left(\lambda_{s}\right)=0$. Hence $\lambda_{s}$ is a simple root of $\rho_{m-1}(\lambda)$ and of $\rho_{m+1}(\lambda)$.

Theorem 5.2. If $p_{m+1}\left(\lambda_{s}\right)=q_{m+1}\left(\lambda_{s}\right)=0(m \geqq 1)$ then $\rho_{m}\left(\lambda_{s}\right)=0=$ $\rho_{m-1}\left(\lambda_{s}\right)$ and there is a common eigenvector $y$ for the corresponding boundary problems (1.1), (1.2), and (1.3) for $m$ and $m-1$ respectively such that $y_{m}\left(\lambda_{s}\right)=y_{m+1}\left(\lambda_{s}\right)=y_{m+2}\left(\lambda_{s}\right)=0$.

Proof. If $p_{m+1}\left(\lambda_{s}\right)=0=q_{m+1}\left(\lambda_{s}\right)$ obviously from (2.2) we have that $\rho_{m}\left(\lambda_{s}\right)=\rho_{m-1}\left(\lambda_{s}\right)=0$. If now $\lambda_{s}$ is a simple root of $\rho_{m}(\lambda)$ then $p_{m+2}\left(\lambda_{s}\right)$ and $q_{m+2}\left(\lambda_{s}\right)$ are not both zero and $y_{n}^{(m)}\left(\lambda_{s}\right)$ defined in (2.3) is a nontrivial eigenvector for the corresponding boundary problem. This vector is also zero at $n=m$ if and only if

$$
\left|\begin{array}{ll}
p_{m}\left(\lambda_{s}\right) & q_{m}\left(\lambda_{s}\right) \\
p_{m+2}\left(\lambda_{s}\right) & q_{m+2}\left(\lambda_{s}\right)
\end{array}\right|=0
$$

This is so by relation (3.5) since $p_{m+1}\left(\lambda_{s}\right)=0=q_{m+1}\left(\lambda_{s}\right)$. Thus $y_{n}^{(m)}\left(\lambda_{s}\right)$ furnishes a common eigenvector for the boundary problem (1.1), (1.2) and (1.3) for $m$ and for $m-1$.

If $\lambda_{s}$ is a double root of $\rho_{m}(\lambda)$ then we have $p_{m+1}\left(\lambda_{s}\right)=q_{m+1}\left(\lambda_{s}\right)=0$ and also $p_{m+2}\left(\lambda_{s}\right)=q_{m+2}\left(\lambda_{s}\right)=0$ by Theorem 4.2. Hence $y_{n}(\lambda)=$ $\alpha p_{n}(\lambda)+\beta q_{n}(\lambda)$ for any constants $\alpha, \beta$ not both 0 provides an eigenvector for the boundary problem (1.1), (1.2) (1.3) for $m$. Since by Lemma 5.1 here $p_{m}\left(\lambda_{s}\right), q_{m}\left(\lambda_{s}\right)$ are not both 0 , the nontrivial vector $y_{n}^{(m-1)}\left(\lambda_{s}\right)=q_{m}\left(\lambda_{s}\right) p_{n}\left(\lambda_{s}\right)-p_{m}\left(\lambda_{s}\right) q_{n}\left(\lambda_{s}\right)$ will then provide a common eigenvector for the boundary problem for $m$ and for $m-1$. 
THEOREM 5.3. If $\rho_{m}\left(\lambda_{s}\right)=0=\rho_{m-1}\left(\lambda_{s}\right)$ then $p_{m+1}\left(\lambda_{s}\right)=0=q_{m+1}\left(\lambda_{s}\right)$ $(m \geqq 1)$.

Proof. Let $\lambda_{s}$ be a simple root of $\rho_{m}(\lambda)$ and of $\rho_{m-1}(\lambda)$, and assume $p_{m+1}\left(\lambda_{s}\right), q_{m+1}\left(\lambda_{s}\right)$ were not both 0 .

Then $y_{n}^{(m-1)}\left(\lambda_{s}\right)$ defined in (2.3) furnishes a nontrivial eigenvector for the boundary problem for $m-1$ satisfying $y_{m}^{(m-1)}\left(\lambda_{s}\right)=y_{m+1}^{(m-1)}\left(\lambda_{s}\right)=$ $y_{m+1}^{(m-1)}\left(\lambda_{s}\right)=0$. Also here from (2.3) we see that $y_{m+2}^{(m-1)}\left(\lambda_{s}\right)=-\rho_{m}\left(\lambda_{s}\right)=0$.

We now obtain a contradiction by the use of relation (3.4) of Lemma 3.3.

We take in relation (3.4) $\lambda=\lambda_{s}, n=m, u_{r}(\lambda)=y_{r}^{(m-1)}(\lambda), v_{r}(\lambda)=$ $y_{r}^{(m-1)}(\lambda)$ and obtain that the left-hand side is positive, and the righthand side is 0 .

We deduce from this contradiction that $y_{n}^{(m-1)}\left(\lambda_{s}\right)$ must be a trivial vector and $p_{m+1}\left(\lambda_{s}\right)=q_{m+1}\left(\lambda_{s}\right)=0$.

If $\lambda_{s}$ is a double root of $\rho_{m}(\lambda)$ or of $\rho_{m-1}(\lambda)$ the result is obviously true by use of Theorem 4.2.

THEOREM 5.4. Between two successive zeros of $\rho_{m-1}(\lambda)(m \geqq 1)$ which are not zeros of $\rho_{m}(\lambda)$ there lies at least one zero of $\rho_{m}(\lambda)$, and vice versa.

Proof. Let $\lambda_{1}, \lambda_{2}$ be consecutive zeros of $\rho_{m-1}(\lambda)$ which are not zeros of $\rho_{m}(\lambda)$. Thus they are both simple zeros of $\rho_{m-1}(\lambda)$ and $p_{m+1}(\lambda), q_{m+1}(\lambda)$ are not both zero at either $\lambda_{1}$ or $\lambda_{2}$ as we see from formula (2.2). $\quad y_{r}^{(m-1)}(\lambda)$ is a nontrivial vector for $\lambda=\lambda_{1}, \lambda_{2} . \quad y_{m}^{(m-1)}(\lambda)=$ $\rho_{m-1}(\lambda)$ is zero at $\lambda_{1}, \lambda_{2}$. Also $\rho_{m-1}^{\prime}\left(\lambda_{1}\right), \rho_{m-1}^{\prime}\left(\lambda_{2}\right)$ have opposite signs. From (2.3), (2.2) we see that $y_{m+2}^{(m-1)}(\lambda)=-\rho_{m}(\lambda)$. We now make use of relation (3.4) for $u_{r}(\lambda)=v_{r}(\lambda)=y_{r}^{(m-1)}(\lambda)$ and $n=m$. We obtain using (2.4), (2.5), (2.6)

$$
\begin{aligned}
\sum_{r=0}^{m} a_{r}\left[y_{r}^{(m-1)}(\lambda)\right]^{2} & =d_{m}\left[y_{m+2}^{(m-1)^{\prime}}(\lambda) y_{m}^{(m-1)}(\lambda)-y_{m+2}^{(m-1)}(\lambda) y_{m}^{(m-1)^{\prime}}(\lambda)\right] \\
& =d_{m}\left[-\rho_{m}^{\prime}(\lambda) \rho_{m-1}(\lambda)+\rho_{m}(\lambda) \rho_{m-1}^{\prime}(\lambda)\right]>0
\end{aligned}
$$

since the left side $>0$ since $m \geqq 1$ and $y_{r}^{(m-1)}(\lambda)$ is not the trivially zero vector. Now $\rho_{m-1}(\lambda)=0$ at $\lambda_{1}, \lambda_{2}$ and $\rho_{m-1}^{\prime}(\lambda)$ has opposite signs at $\lambda_{1}, \lambda_{2}$. Hence we deduce that $\rho_{m}(\lambda)$ has opposite signs at $\lambda_{1}, \lambda_{2}$ and hence $\rho_{m}(\lambda)$ has at least one zero and in general an odd number of zeros between $\lambda_{1}$ and $\lambda_{2}$.

In the case of two consecutive zeros $\lambda_{1}, \lambda_{2}$ of $\rho_{m}(\lambda)$ which are not zeros of $\rho_{m-1}(\lambda)$ and hence are simple zeros of $\rho_{m}(\lambda)$, with $p_{m+1}(\lambda)$, $q_{m+1}(\lambda)$ not both equal to zero at either $\lambda_{1}$ or $\lambda_{2}$, we have similarly that $\rho_{m}^{\prime}(\lambda)$ has opposite signs at $\lambda=\lambda_{1}, \lambda_{2}$. From the same relation obtained above we then deduce that $\rho_{m-1}(\lambda)$ has opposite signs at 
$\lambda_{1}, \lambda_{2}$ and hence $\rho_{m-1}(\lambda)$ has at least one zero, and in general an odd number of zeros, between $\lambda_{1}$ and $\lambda_{2}$.

6. Orthogonality and expansion theorems. We now obtain orthogonality relations for the eigenvectors of the boundary problem (1.1), (1.2), (1.3) and corresponding expansion theorems.

We consider the set of eigenvalues $\lambda_{i}(i=0,1, \cdots, m)$ of the boundary problem (1.1), (1.2), (1.3), roots of the equation $\rho_{m}(\lambda)=0$ where $\rho_{m}(\lambda)$ is defined in (2.2), where multiple eigenvalues are counted according to their multiplicity and written a corresponding number of times. We note that the eigenvalues $\lambda_{i}$ are all real by Theorem 4.1 and only double eigenvalues at most can occur by Theorem 4.3.

We take

$$
\lambda_{0} \leqq \lambda_{1} \leqq \lambda_{2} \leqq \cdots \leqq \lambda_{m} .
$$

We obtain with the aid of Theorem 4.2 a corresponding set of $m+1$ mutually orthogonal eigenvectors. The form will vary in various cases and according to whether $\lambda_{i}$ is a simple or double eigenvalue.

We consider firstly the case where $\lambda_{i}$ is a simple root of equation $\rho_{m}(\lambda)=0$ and $p_{m+2}\left(\lambda_{i}\right)+h p_{m}\left(\lambda_{i}\right), q_{m+2}\left(\lambda_{i}\right)+h q_{m}\left(\lambda_{i}\right)$ are not both 0 . Then as seen in $\S 2$ the vector $y_{r}^{(m)}\left(\lambda_{i}\right)$ defined by (2.3) furnishes a nontrivial real eigenvector of the boundary problem (1.1), (1.2), (1.3). Consider a similar root $\lambda_{j} \neq \lambda_{i}$ and corresponding eigenvector $y_{r}^{(m)}\left(\lambda_{j}\right)$.

We now make use of relation (3.1) of Lemma 3.1 with $\lambda=\lambda_{i}$, $\mu=\lambda_{j}$ and $u_{r}(\lambda)=y_{r}^{(m)}\left(\lambda_{i}\right), v_{r}(\mu)=y_{r}^{(m)}\left(\lambda_{j}\right)$ and obtain

$$
\left(\lambda_{i}-\lambda_{j}\right) \sum_{r=0}^{m} a_{r} y_{r}^{(m)}\left(\lambda_{i}\right) y_{r}^{(m)}\left(\lambda_{j}\right)=0 .
$$

Using $\lambda_{i} \neq \lambda_{j}$ we obtain the orthogonality relation

$$
\sum_{r=0}^{m} a_{r} y_{r}^{(m)}\left(\lambda_{i}\right) y_{r}^{(m)}\left(\lambda_{j}\right)=0 .
$$

We see that (6.2) is trivially true if $\lambda_{i}$ or $\lambda_{j}$ is not a simple root using Theorem 4.2 or if $p_{m+2}(\lambda)+h p_{m}(\lambda)=0, q_{m+2}(\lambda)+h q_{m}(\lambda)=0$ for $\lambda=\lambda_{i}$ or $\lambda=\lambda_{j}$.

Similarly if $\lambda_{i}$ is a simple root of $\rho_{m}(\lambda)=0$ and

$$
\begin{aligned}
& p_{m+1}\left(\lambda_{i}\right)+k\left[c_{m} p_{m}\left(\lambda_{i}\right)+d_{m-1} p_{m-1}\left(\lambda_{i}\right)\right], \\
& q_{m+1}\left(\lambda_{i}\right)+k\left[c_{m} q_{m}\left(\lambda_{i}\right)+d_{m-1} q_{m-1}\left(\lambda_{i}\right)\right]
\end{aligned}
$$

are not both 0 , we can use the vector $z_{r}^{(m)}\left(\lambda_{i}\right)$ defined by (2.7) as a nontrivial real eigenvector. Then relation (3.1) furnishes similar orthogonality relations.

In the case of a double root $\lambda_{i}=\lambda_{i+1}$ of $\rho_{m}(\lambda)=0$ we obtain by 
Theorem 4.2 two linearly independent real eigenvectors $p_{r}\left(\lambda_{i}\right), q_{r}\left(\lambda_{i}\right)$ and from these we can form two orthogonal eigenvectors, for example by taking

$$
\widetilde{p}_{r}=p_{r}, \widetilde{q}_{r}=q_{r}-\frac{\sum_{s=0}^{m} a_{s} p_{s} q_{s}}{\sum_{s=0}^{m} a_{s} p_{s}^{2}} p_{r} .
$$

We hence can form for a given boundary problem (1.1), (1.2), (1.3) for given $m \geqq 0$ a set of $m+1$ mutually orthogonal real eigenvectors corresponding to the set of eigenvalues $\lambda_{0}, \lambda_{1}, \cdots, \lambda_{m}$.

We let $\left\{w_{i n}\right\}_{n=-2}^{m+2}(i=0,1, \cdots, m)$ be defined as the normalized nontrivial real eigenvector corresponding to the eigenvalue $\lambda_{i}$ which is formed, in the case that $\lambda_{i}$ is a simple eigenvalue with $p_{m+2}\left(\lambda_{i}\right)+$ $h p_{m}\left(\lambda_{i}\right), q_{m+2}\left(\lambda_{i}\right)+h q_{m}\left(\lambda_{i}\right)$ not both zero, from $y_{n}^{(m)}\left(\lambda_{i}\right)$, while, if $\lambda_{i}$ is a simple eigenvalue with $p_{m+2}\left(\lambda_{i}\right)+h p_{m}\left(\lambda_{i}\right), q_{m+2}\left(\lambda_{i}\right)+h q_{m}\left(\lambda_{i}\right)$ both zero, from $z_{n}^{(m)}\left(\lambda_{i}\right)$, and, in the case that $\lambda_{i}=\lambda_{i+1}$ is a double eigenvalue, from $\widetilde{p}_{n}\left(\lambda_{i}\right)$ and $\widetilde{q}_{n}\left(\lambda_{i}\right)$ respectively, by scaling in each case by division by a positive factor so that $w_{i 0}^{2}+w_{i 1}^{2}=1$.

For the real eigenvectors $\left\{w_{i n}\right\}_{n=-2}^{m+2}(i=0,1, \cdots, m)$ we define

$$
\beta_{i}=\sum_{n=0}^{m} a_{n} w_{i n}^{2}
$$

The vectors $\left\{w_{i n}\right\}$ satisfy the orthogonality relations

$$
\sum_{n=0}^{m} a_{n} w_{i n} w_{j n}=\beta_{i} \delta_{i j} .
$$

We now replace the vectors $\left\{w_{i n}\right\}$ by the normalized eigenvectors

$$
\left\{x_{i n}\right\}=\left\{w_{i n} \beta_{i}^{-1 / 2}\right\} \text {. }
$$

Then we have the following theorem:

THEOREM 6.1. The boundary problem (1.1), (1.2), (1.3) for given $m, h, k$ has a set of $m+1$ real mutually orthogonal eigenvectors $\left\{x_{i n}\right\}_{n=-2}^{m+2}(i=0,1, \cdots, m)$ corresponding to the real eigenvalues $\lambda_{i}$ $(0 \leqq i \leqq m)$ where $\lambda_{0} \leqq \lambda_{1} \leqq \lambda_{2} \leqq \cdots \leqq \lambda_{m}$, which satisfy the orthogonality relations

$$
\sum_{n=0}^{m} a_{n} x_{i n} x_{j n}=\delta_{i j} \quad(0 \leqq i, j \leqq m) .
$$

We now obtain an expansion theorem for an arbitrary sequence in terms of the eigenvectors and a corresponding completeness theorem:

THEOREM 6.2. If $\left\{f_{n}\right\}_{n=0}^{m}$ is an arbitrary vector we define 


$$
v_{i}=\sum_{n=0}^{m} a_{n} f_{n} x_{i n} \quad(i=0,1, \cdots, m)
$$

where the $\left\{x_{i n}\right\}_{n=-2}^{m+2}(i=0,1, \cdots, m)$ are the real orthogonalized eigenvectors of the previous theorem. Then we have the expansion

$$
f_{n}=\sum_{i=0}^{m} v_{i} x_{i n} \quad(n=0,1, \cdots, m) .
$$

Also the Parseval equality or completeness relation holds

$$
\sum_{i=0}^{m}\left|v_{i}\right|^{2}=\sum_{n=0}^{m} a_{n}\left|f_{n}\right|^{2}
$$

Proof. Let

$$
f_{n}=\sum_{i=0}^{m} \alpha_{i} x_{i n} \quad(n=0,1, \cdots, m) .
$$

This expansion is possible since we have $m+1$ linearly independent eigenvectors $\left\{x_{i n}\right\}_{n=0}^{m}(i=0,1, \cdots, m)$ in the $m+1$ dimensional vector space by (6.7).

Multiply (6.11) by $a_{n} x_{j n}$ and sum over $n$ from 0 to $m$. We obtain

$$
\begin{aligned}
\sum_{n=0}^{m} a_{n} f_{n} x_{j n} & =\sum_{n=0}^{m} a_{n} x_{j n}\left(\sum_{i=0}^{m} \alpha_{i} x_{i n}\right) \\
& =\sum_{i=0}^{m} \alpha_{i}\left(\sum_{n=0}^{m} a_{n} x_{j n} x_{i n}\right) \\
& =\sum_{i=0}^{m} \alpha_{i} \delta_{i j} \\
& =\alpha_{j}
\end{aligned}
$$

using the orthogonality relations (6.7).

Hence from (6.8) we see that

$$
a_{j}=v_{j}
$$

and from (6.11) we have (6.9).

To prove (6.10) we have, using (6.9), (6.7),

$$
\begin{aligned}
\sum_{n=0}^{m} a_{n}\left|f_{n}\right|^{2} & =\sum_{n=0}^{m} a_{n}\left(\sum_{i=0}^{m} v_{i} x_{i n} \sum_{j=0}^{m} \bar{v}_{j} x_{j n}\right) \\
& =\sum_{i, j=0}^{m} v_{i} \bar{v}_{j}\left(\sum_{n=0}^{m} a_{n} x_{i n} x_{j n}\right) \\
& =\sum_{i, j=0}^{m} v_{i} \bar{v}_{j} \delta_{i j} \\
& =\sum_{i=0}^{m}\left|v_{i}\right|^{2} .
\end{aligned}
$$

Hence the proof of the theorem is complete.

We also have dual orthogonality relations: 
THEOREM 6.3. For the real eigenvectors $\left\{x_{i n}\right\}_{n=-2}^{m+2}(i=0,1, \cdots, m)$ of Theorem 6.1 we have the dual orthogonality relations

$$
\sum_{i=0}^{m} x_{i r} x_{i s}=a_{r}^{-1} \delta_{r s} \quad(0 \leqq r, s \leqq m) .
$$

Proof. Substituting (6.7) in (6.8) we obtain

$$
\begin{aligned}
f_{r} & =\sum_{i=0}^{m} x_{i r}\left(\sum_{s=0}^{m} a_{s} f_{s} x_{i s}\right) \\
& =\sum_{s=0}^{m} a_{s} f_{s}\left(\sum_{\imath=0}^{m} x_{i r} x_{i s}\right) .
\end{aligned}
$$

Since the vector $\left\{f_{n}\right\}_{n=0}^{m}$ is arbitrary we deduce

$$
\sum_{i=0}^{m} x_{i r} x_{i s}=a_{r}^{-1} \delta_{r s} .
$$

We derive the dual expansion theorem:

THEOREM 6.4. Defining for an arbitrary vector $\left\{v_{i}\right\}_{i=0}^{m}$ the $f_{n}(n=$ $0,1, \cdots, m)$ by (6.9), the expansion theorem (6.8) is true.

Proof. The vectors $\left\{x_{i n}\right\}_{i=0}^{m}(n=0,1, \cdots, m)$ for different $n$ are orthogonal by (6.12) and hence linearly independent. Hence we can express an arbitrary vector $\left\{v_{i}\right\}_{i=0}^{m}$ in terms of them. The proof now follows by use of (6.12) analogously to the proof of Theorem 6.2.

7. Spectral functions. The preceding orthogonality relations and expansion theorems can be expressed in terms of a spectral function defined for the boundary problem (1.1), (1.2) (1.3). This is especially useful when later considering the boundary problem in the infinite case $m \rightarrow \infty$ and the corresponding expansion theorems.

We define the row matrix of functions of $\lambda$

$$
Y_{n}(\lambda)=\left[p_{n}(\lambda), q_{n}(\lambda)\right] \quad(n \geqq-2)
$$

satisfying the recurrence relations

$$
d_{n} Y_{n+2}+c_{n} Y_{n+1}+\left(b_{n}-\lambda a_{n}\right) Y_{n}+c_{n-1} Y_{n-1}+d_{n-2} Y_{n-2}=0 \quad(n \geqq 0)
$$

with the initial conditions

$$
Y_{-2}(\lambda)=(0,0), Y_{-1}(\lambda)=(0,0), Y_{0}(\lambda)=(1,0), Y_{1}(\lambda)=(0,1) .
$$

The vectors $\left\{x_{i n}\right\}_{n=-2}^{m+2}(i=0,1, \cdots, m)$ defined in $\S 6$ may be expressed by

$$
\begin{aligned}
x_{i n} & =x_{i 0} p_{n}\left(\lambda_{i}\right)+x_{i 1} q_{n}\left(\lambda_{i}\right) \\
& =Y_{n}\left(\lambda_{i}\right) u_{i}
\end{aligned}
$$

where the vectors $u_{i}(i=0,1, \cdots, m)$ are defined by 


$$
u_{i}=\left(\begin{array}{l}
x_{i 0} \\
x_{i 1}
\end{array}\right) .
$$

Then, denoting by an asterisk the complex conjugate transpose of a matrix, the orthogonality relations (6.7) can be written in the form

$$
\sum_{n=0}^{m} u_{i}^{*} Y_{n}^{*}\left(\lambda_{i}\right) a_{n} Y_{n}\left(\lambda_{j}\right) u_{j}=\delta_{i j} \quad(0 \leqq i, j \leqq m) .
$$

The dual orthogonality relations (6.12) become

$$
\sum_{i=0}^{m} Y_{r}\left(\lambda_{i}\right) u_{i} u_{i}^{*} Y_{s}^{*}\left(\lambda_{i}\right)=a_{r}^{-1} \delta_{r s} \quad(0 \leqq r, s \leqq m) .
$$

These may be written in the form

$$
\int_{-\infty}^{\infty} Y_{r}(\lambda) d \tau_{m, h, k}(\lambda) Y_{s}^{*}(\lambda)=a_{r}^{-1} \delta_{r s} \quad(0 \leqq r, s \leqq m)
$$

where the two-dimensional spectral function $\tau_{m, h, k}(\lambda)$ is defined by

$$
\begin{aligned}
\tau_{m, h, k}(\lambda) & =\sum_{0<\lambda_{i} \leqq \lambda} u_{i} u_{i}^{*} \quad(\lambda \geqq 0) \\
& =-\sum_{\lambda<\lambda_{i} \leqq 0} u_{i} u_{i}^{*} \quad(\lambda<0)
\end{aligned}
$$

where $\lambda_{i}(i=0,1, \cdots, m)$ are the eigenvalues of the boundary problem (1.1), (1.2), (1.3) satisfying (6.1).

Thus the spectral function in this finite case $0 \leqq m<\infty$ is a right-continuous matrix step-function with finite jumps at simple eigenvalues $\lambda_{i}$ of amount $u_{i} u_{i}^{*}$ and at double eigenvalues $\lambda_{i}=\lambda_{i+1}$ of amount $u_{i} u_{i}^{*}+u_{i+1} u_{i+1}^{*}$.

The expansion and completeness theorem Theorem 6.2 of $\S 6$ can now be rewritten as follows:

THEOREM 7.1. If $\left\{f_{n}\right\}(n=0,1, \cdots, m)$ is an arbitrary vector we define the vector function

$$
g(\lambda)=\sum_{n=0}^{m} Y_{n}^{*}(\lambda) a_{n} f_{n} .
$$

Then we have the expansion

$$
\begin{gathered}
f_{n}=\sum_{i=0}^{m} Y_{n}\left(\lambda_{i}\right) u_{i} u_{i}^{*} g\left(\lambda_{i}\right) \\
=\int_{-\infty}^{\infty} Y_{n}(\lambda) d \tau_{m, h, k}(\lambda) g(\lambda) \quad(0 \leqq n \leqq m) .
\end{gathered}
$$

Also the Parseval equality or completeness relation holds

$$
\begin{aligned}
\sum_{n=0}^{m} a_{n}\left|f_{n}\right|^{2} & =\sum_{i=0}^{m}\left|g^{*}\left(\lambda_{i}\right) u_{i}\right|^{2} \\
& =\int_{-\infty}^{\infty} g^{*}(\lambda) d \tau_{m, h, k}(\lambda) g(\lambda) .
\end{aligned}
$$


Proof. We use in Theorem 6.2 relation (7.4) and note that the vector $\left\{v_{i}\right\}_{i=0}^{m}$ of (6.8) is given by $v_{i}=u_{i}^{*} g\left(\lambda_{i}\right)$.

8. Inhomogeneous boundary problems. We now consider in this section the solution of the boundary problem for the inhomogeneous recurrence relations

$$
\begin{aligned}
d_{n} y_{n+2} & +c_{n} y_{n+1}+\left(b_{n}-\lambda a_{n}\right) y_{n}+c_{n-1} y_{n-1} \\
& +d_{n-2} y_{n-2}=a_{n} f_{n} \quad(0 \leqq n \leqq m)
\end{aligned}
$$

where the coefficients $a_{n}, b_{n}, c_{n}, d_{n}$ are real, $a_{n}, d_{n}>0, \lambda$ is a complex parameter and where $\left\{f_{n}\right\}(0 \leqq n \leqq m)$ is a given complex vector, with the boundary conditions

$$
y_{-2}=0=y_{-1}
$$

and

$$
y_{m+1}+k\left[c_{m} y_{m}+d_{m-1} y_{m-1}\right]=0, y_{m+2}+h y_{m}=0
$$

where $h, k$ are real constants and $m \geqq 0$.

The solution is given in terms of a Green's functions for the recurrence relations (1.1).

We first obtain some fundamental lemmas:

LEMMA 8.1. For $m \geqq-1, n \geqq-1$

$$
\begin{aligned}
{[p \bar{r}]_{m}|q s|_{n} } & +[q \bar{s}]_{m}|p r|_{n}-[p \bar{q}]_{m}|r s|_{m}-[p \bar{s}]_{m}|q r|_{n}-[q \bar{r}]_{m}|p s|_{n} \\
& -[r \bar{s}]_{m}|p q|_{n}=0
\end{aligned}
$$

where $[u v]_{n}$ is defined in (3.1) and $|u v|_{n}$ in (2.2).

In particular

$$
d_{-2}|q s|_{n}+d_{-1}|p r|_{n}-c_{-1}|q r|_{n}=0 \quad(n \geqq-1) .
$$

Proof. Using (3.6) we have

$$
[p \bar{r}]_{m}|q s|_{n}=[p \bar{r}]_{n}|q s|_{n} .
$$

Adding this and the similar relations for the terms on the left side of (8.4) we obtain the expansion in terms of second order minors of the sum of three fourth order determinants which sum to zero.

(8.5) is obtained from (8.4) by use of relations (3.7). We also have

LEMMA 8.2. For $m \geqq-1, n \geqq-2$

$$
\begin{aligned}
{[p \bar{r}]_{m}(q s)_{n} } & +[q \bar{s}]_{m}(p r)_{n}-[p \bar{q}]_{m}(r s)_{n}-[p \bar{s}]_{m}(q r)_{n} \\
& -[q \bar{r}]_{m}(p s)_{n}-[r \bar{s}]_{m}(p q)_{n}=d_{-1} d_{-2}
\end{aligned}
$$


where $[u v]_{n}$ is defined in (3.1) and $(u v)_{n}$ is defined by

$$
(u v)_{n}=d_{n}\left(u_{n+2} v_{n}-v_{n+2} u_{n}\right) .
$$

In particular

$$
d_{-2}(q s)_{n}+d_{-1}(p r)_{n}-c_{-1}(q r)_{n}=d_{-1} d_{-2} . \quad(n \geqq-2)
$$

Proof. Using (3.6) we have

$$
[p \bar{r}]_{m}(q s)_{n}=[p \bar{r}]_{n}(q s)_{n} .
$$

Adding this and similar relations for the terms on the left side of (8.6) we obtain the expansion in terms of second order minors of the sum of three fourth order determinants of which two are equal to zero and the third is equal to $D_{n}$ defined by

$$
D_{n}=d_{n} d_{n-1}\left|\begin{array}{llll}
p_{n-1} & q_{n-1} & r_{n-1} & s_{n-1} \\
p_{n} & q_{n} & r_{n} & s_{n} \\
p_{n+1} & q_{n+1} & r_{n+1} & s_{n+1} \\
p_{n+2} & q_{n+2} & r_{n+2} & s_{n+2}
\end{array}\right| . \quad(n \geqq-1)
$$

Now substituting for $p_{n+2}, q_{n+2}, r_{n+2}, s_{n+2}$ from the recurrence relations (1.1) in $D_{n}$ we obtain $D_{n}=D_{n-1}(n \geqq 0)$, and by induction, using $D_{-1}=d_{-2} d_{-1}$,

$$
D_{n}=d_{-2} d_{-1} \quad(n \geqq-1)
$$

and thus we have the result (8.6). We obtain (8.8) from (8.6) by use of relations (3.6), (3.7).

We now define two solutions $\psi_{i}^{(m)}(\lambda)(i=1,2)$ of the recurrence relations (1.1) in terms of the fundamental solutions $p, q, r, s$ defined in (2.1). They are characterized by the following lemma:

Lemma 8.3. The solutions $\psi_{i}^{(m)}(\lambda)(i=1,2)$ of the recurrence relations (1.1) satisfying also boundary conditions (1.3) and the relations

$$
\left[\psi_{1} \bar{p}\right]=1,\left[\psi_{1} \bar{q}\right]=0,\left[\psi_{2} \bar{p}\right]=0,\left[\psi_{2} \bar{q}\right]=1,
$$

where the form $[u \bar{v}]$ for two solutions of (1.1) is defined in (3.1), (3.6), are given, for $\lambda$ not an eigenvalue of the boundary problem (1.1), (1.2), (1.3), by

$$
\begin{aligned}
\psi_{1 n}^{(m)}(\lambda)= & -\frac{1}{d_{-2}}\left[\frac{|q r|_{m}}{|p q|_{m}} p_{n}-\frac{|p r|_{m}}{|p q|_{m}} q_{n}+r_{n}\right] \\
\psi_{2 n}^{(m)}(\lambda)= & \frac{c_{-1}}{d_{-1} d_{-2}}\left[\frac{|q r|_{m}}{|p q|_{m}} p_{n}-\frac{|p r|_{m}}{|p q|_{m}} q_{n}+r_{n}\right] \\
& -\frac{1}{d_{-1}}\left[\frac{|q s|_{m}}{|p q|_{m}} p_{n}-\frac{|p s|_{m}}{|p q|_{m}} q_{n}+s_{n}\right](-2 \leqq n \leqq m+2)
\end{aligned}
$$


where the polynomials $|p q|_{m}$ are defined in (2.2).

The $\psi_{i}^{(m)}(\lambda)$ satisfy in addition

$$
\left[\psi_{1} \bar{\psi}_{2}\right]=0 \text {. }
$$

Proof. We attempt to solve the equations (1.1), (1.3), (8.11) by setting $y_{n}(\lambda)=\alpha p_{n}(\lambda)+\beta q_{n}(\lambda)+\gamma r_{n}(\lambda)+\delta s_{n}(\lambda)$ where $\alpha, \beta, \gamma, \delta$ are undetermined constants.

We then use first condition (1.3) and then conditions (8.11) to derive the solutions $\psi_{1}^{(m)}(\lambda), \psi_{2}^{(m)}(\lambda)$ in the form given by (8.12).

To prove (8.13) we use (8.12) and (3.7) and hence have that

$$
\left[\psi_{1} \bar{\psi}_{2}\right]=\frac{1}{|p q|_{m}}\left[\frac{c_{-1}}{d_{-1} d_{-2}}|q r|_{m}-\frac{1}{d_{-2}}|p r|_{m}-\frac{1}{d_{-1}}|q s|_{m}\right]=0
$$

by (8.5) of Lemma 8.1.

We now demonstrate the following lemma:

LEMMA 8.4.

$$
\begin{aligned}
\psi_{1 t}^{(m)} p_{n}+\psi_{2 t}^{(m)} q_{n}-\psi_{1 n}^{(m)} p_{t}-\psi_{2 n}^{(m)} q_{t} \\
\quad=\frac{1}{d_{-1} d_{-2}}\left[d_{-2}\left|\begin{array}{ll}
q_{t} & s_{t} \\
q_{n} & s_{n}
\end{array}\right|+d_{-1}\left|\begin{array}{ll}
p_{t} & r_{t} \\
p_{n} & r_{n}
\end{array}\right|-c_{-1}\left|\begin{array}{ll}
q_{t} & r_{t} \\
q_{n} & r_{n}
\end{array}\right|\right]
\end{aligned}
$$

for $-2 \leqq t, n \leqq m+2$.

Proof. We use formula (8.12) and relation (8.5) of Lemma 8.1. We have

$$
\begin{aligned}
\psi_{1 t}^{(m)} p_{n} & +\psi_{2 t}^{(m)} q_{n} \\
= & -\frac{1}{d_{-2}}\left(\frac{|q r|_{m}}{|p q|_{m}} p_{t}-\frac{|p r|_{m}}{|p q|_{m}} q_{t}+r_{t}\right) p_{n}+\frac{c_{-1}}{d_{-1} d_{-2}}\left(\frac{|q r|_{m}}{|p q|_{m}} p_{t}\right. \\
& \left.-\frac{|p r|_{m}}{|p q|_{m}} q_{t}+r_{t}\right) q_{n}-\frac{1}{d_{-1}}\left(\frac{|q s|_{m}}{|p q|_{m}} p_{t}-\frac{|p s|_{m}}{|p q|_{m}} q_{t}+s_{t}\right) q_{n} \\
= & p_{t} \frac{1}{|p q|_{m}} \frac{1}{d_{-1} d_{-2}}\left[-d_{-1}|q r|_{m} p_{n}+c_{-1}|q r|_{m} q_{n}-d_{-2}|q s|_{m} q_{n}\right] \\
& +q_{t} \frac{1}{|p q|_{m}} \frac{1}{d_{-1} d_{-2}}\left[d_{-1}|p r|_{m} p_{n}-c_{-1}|p r|_{m} q_{n}+d_{-2}|p s|_{m} q_{n}\right] \\
& +\frac{1}{d_{-1} d_{-2}}\left[-d_{-1} r_{t} p_{n}+c_{-1} r_{t} q_{n}-d_{-2} s_{t} q_{n}\right] \\
= & \frac{1}{|p q|_{m}} \frac{1}{d_{-1} d_{-2}}\left\{p _ { t } \left[d_{-1}|p r|_{m} q_{n}-d_{-1}|q r|_{m} p_{n}+q_{t}\left[-d_{-2}|q s|_{m} p_{n}\right.\right.\right. \\
& \left.\left.+c_{-1}|q r|_{m} p_{n}-c_{-1}|p r|_{m} q_{n}+d_{-2}|p s|_{m} q_{n}\right]\right\} \\
& +\frac{1}{d_{-1} d_{-2}}\left[-d_{-1} r_{t} p_{n}+c_{-1} r_{t} q_{n}-d_{-2} s_{t} q_{n}\right] .
\end{aligned}
$$


Hence

$$
\begin{aligned}
\psi_{1 t}^{(m)} p_{n}+\psi_{2 t}^{(m)} q_{n}-\psi_{1 n}^{(m)} p_{t}-\psi_{2 n}^{(m)} q_{t} \\
=\frac{1}{d_{-1} d_{-2}} \frac{1}{|p q|_{m}}\left(p_{t} q_{n}-p_{n} q_{t}\right)\left[d_{-1}|p r|_{m}+d_{-2}|q s|_{m}-c_{-1}|q r|_{m}\right] \\
\quad+\frac{1}{d_{-1} d_{-2}}\left[d_{-2}\left|\begin{array}{cc}
q_{t} & s_{t} \\
q_{n} & s_{n}
\end{array}\right|+d_{-1}\left|\begin{array}{cc}
p_{t} & r_{t} \\
p_{n} & r_{n}
\end{array}\right|-c_{-1}\left|\begin{array}{cc}
q_{t} & r_{t} \\
q_{n} & s_{n}
\end{array}\right|\right] \\
=\frac{1}{d_{-1} d_{-2}}\left[d_{-2}\left|\begin{array}{ll}
q_{t} & s_{t} \\
q_{n} & s_{n}
\end{array}\right|+d_{-1}\left|\begin{array}{cc}
p_{t} & r_{t} \\
p_{n} & r_{n}
\end{array}\right|-c_{-1}\left|\begin{array}{cc}
q_{t} & r_{t} \\
q_{n} & r_{n}
\end{array}\right|\right]
\end{aligned}
$$

using relation (8.5).

We now can obtain the following theorem:

THEOREM 8.1. The solution of the inhomogeneous boundary problem (8.1), (8.2), (8.3) for $\lambda$ not an eigenvalue of the corresponding homogeneous boundary problem (1.1), (1.2), (1.3) is unique and is given by

$$
y_{r}(\lambda)=\sum_{i=0}^{m} g_{r i}^{(m)}(\lambda) f_{i} \quad(-2 \leqq r \leqq m+2)
$$

where the Green's function $g_{r i}^{(m)}(\lambda)$ is given by

$$
\begin{aligned}
g_{r r}^{(m)}(\lambda) & =a_{i} \psi_{\psi_{i}}^{(m)}(\lambda) p_{r}(\lambda)+a_{i} \psi_{2 i}^{(m)}(\lambda) q_{r}(\lambda) & & (r \leqq i) \\
& =a_{i} p_{i}(\lambda) \psi_{1 r}^{(m)}(\lambda)+a_{i} q_{i}(\lambda) \psi_{2 r}^{(m)}(\lambda) & & (r \geqq i)
\end{aligned}
$$

for $0 \leqq i \leqq m,-2 \leqq r \leqq m+2$, and where the solutions of (1.1) $p_{n}(\lambda), q_{n}(\lambda)$ and $\psi_{i n}^{(m)}(\lambda)(i=1,2)$ are defined in (2.1) and (8.12) respectively.

The solution $y(\lambda)=\left\{y_{r}(\lambda)\right\}(-2 \leqq r \leqq m+2)$ for $\operatorname{Im} \lambda \neq 0$ satisfies

$$
\|y\|_{m} \leqq|\operatorname{Im} \lambda|^{-1}|| f \|_{m}
$$

where

$$
\|y\|_{m}=\left(\sum_{n=0}^{m} a_{n}\left|y_{n}\right|^{2}\right)^{1 / 2}
$$

The Green's function $g_{n \iota}^{(m)}(\lambda)$ for $\operatorname{Im} \lambda \neq 0$ is analytic in $\lambda$ and has the following properties:

$$
\text { (i) } a_{i}^{-1} g_{n \imath}^{(m)}(\lambda)=a_{n}^{-1} g_{i n}^{(m)}(\lambda)
$$

$$
(0 \leqq n, i \leqq m)
$$

$$
\text { (ii) } \sum_{n=0}^{m} a_{n}\left|g_{n i}^{(m)}(\lambda)\right|^{2} \leqq|\operatorname{Im} \lambda|^{-2} a_{i}
$$

$$
(0 \leqq i \leqq m)
$$

(iii) $\left|g_{n i}^{(m)}(\lambda)\right| \leqq|\operatorname{Im} \lambda|^{-1} a_{i}^{1 / 2} a_{n}^{-1 / 2}$

$(0 \leqq n, i \leqq m)$

$$
\text { (iv) } \sum_{n=0}^{m} a_{n}^{-1}\left|g_{i n}^{(m)}(\lambda)\right|^{2} \leqq|\operatorname{Im} \lambda|^{-2} a_{i}^{-1} \quad(0 \leqq i \leqq m)
$$

(v) $d_{n} g_{n+2 i}^{(m)}+c_{n} g_{n+1 i}^{(m)}+b_{n} g_{n \imath}^{(m)}+c_{n-1} g_{n-1 i}^{(m)}+d_{n-2} g_{n-2 i}^{(m)}$

$$
=\lambda a_{n} g_{n i}^{(m)}+a_{i} \delta_{n i} \quad(0 \leqq n, i \leqq m)
$$




$$
\begin{aligned}
g_{-2 i}^{(m)}=g_{-1 i}^{(m)}=0 & (0 \leqq i \leqq m), \\
g_{m+1 i}+k\left[c_{m} g_{m i}+d_{m-1} g_{m-1 i}\right]=0 & \\
g_{m+2 i}+h g_{m i}=0 & (0 \leqq i \leqq m) .
\end{aligned}
$$

Proof. The solution of the boundary problem (8.1), (8.2), (8.3) in the form (8.15), (8.16) can be obtained by a method of variation of parameters. We seek to find the Green's function $\left\{g_{r i}^{(m)}(\lambda)\right\}_{r=-2}^{m+2}$ for fixed $i$ satisfying $0 \leqq i \leqq m$ given by the solution $y=\left\{y_{n}\right\}_{-2}^{m+2}$ of (8.1), (8.2), (8.3) taking for $\left\{f_{n}\right\}_{0}^{m}$ the vector $\left\{\delta_{n i}\right\}_{n=0}^{m}$. The solution of (8.1), (8.2), (8.3) for an arbitrary vector $\left\{f_{n}\right\}_{0}^{m}$ will then be given by (8.15) by superposition.

We set

$$
y_{n}(\lambda)=\alpha_{n} p_{n}(\lambda)+\beta_{n} q_{n}(\lambda)+\gamma_{n} r_{n}(\lambda)+\delta_{n} s_{n}(\lambda) \quad(n \geqq-2)
$$

where $\alpha_{n}, \beta_{n}, \gamma_{n}, \delta_{n}$ are complex constants and $p_{n}(\lambda), q_{n}(\lambda), r_{n}(\lambda), s_{n}(\lambda)$ are the fundamental set of solutions of the corresponding homogeneous boundary problem (1.1), (1.2), (1.3) given by (2.1).

Substituting in (8.1) from (8.26) and using the recurrence relations (1.1) satisfied by $p_{n}, q_{n}, r_{n}, s_{n}$ we obtain the equations (if $a_{i}=1$ )

$$
\begin{aligned}
& d_{n}\left[\left(\alpha_{n+2}-\alpha_{n}\right) p_{n+2}+\left(\beta_{n+2}-\beta_{n}\right) q_{n+2}+\left(\gamma_{n+2}-\gamma_{n}\right) r_{n+2}\right. \\
& \left.\quad+\left(\delta_{n+2}-\delta_{n}\right) s_{n+2}\right]+c_{n}\left[\left(\alpha_{n+1}-\alpha_{n}\right) p_{n+1}+\left(\beta_{n+1}-\beta_{n}\right) q_{n+1}\right. \\
& \left.\quad+\left(\gamma_{n+1}-\gamma_{n}\right) r_{n+1}+\left(\delta_{n+1}-\delta_{n}\right) s_{n+1}\right]+c_{n-1}\left[\left(\alpha_{n-1}-\alpha_{n}\right) p_{n-1}\right. \\
& \left.\quad+\left(\beta_{n-1}-\beta_{n}\right) q_{n-1}+\left(\gamma_{n-1}-\gamma_{n}\right) r_{n-1}+\left(\delta_{n-1}-\delta_{n}\right) s_{n-1}\right] \\
& \quad+d_{n-2}\left[\left(\alpha_{n-2}-\alpha_{n}\right) p_{n-2}+\left(\beta_{n-2}-\beta_{n}\right) q_{n-2}+\left(\gamma_{n-2}-\gamma_{n}\right) r_{n-2}\right. \\
& \left.\quad+\left(\delta_{n-2}-\delta_{n}\right) s_{n-2}\right]=\delta_{n i} \quad(0 \leqq n \leqq m) .
\end{aligned}
$$

We satisfy equation (8.27) for $0 \leqq n \leqq i-2$ and for $i+3 \leqq n \leqq m$ by choosing

$$
\begin{gathered}
\alpha_{m+2}=\cdots=\alpha_{i+2}=\alpha_{i+1} \neq \alpha_{i}=\alpha_{i-1}=\cdots=\alpha_{-2} \\
\beta_{m+2}=\cdots=\beta_{i+2}=\beta_{i+1} \neq \beta_{i}=\beta_{i-1}=\cdots=\beta_{-2} \\
\gamma_{m+2}=\cdots=\gamma_{i+2}=\gamma_{i+1} \neq \gamma_{i}=\gamma_{i-1}=\cdots=\gamma_{-2} \\
\delta_{m+2}=\cdots=\delta_{i+2}=\delta_{i+1} \neq \delta_{i}=\delta_{i-1}=\cdots=\delta_{-2}
\end{gathered}
$$

and setting

$$
\widehat{\alpha}=\alpha_{i+1}-\alpha_{i}, \widehat{\beta}=\beta_{i+1}-\beta_{i}, \hat{\gamma}=\gamma_{i+1}-\gamma_{i}, \widehat{\delta}=\delta_{i+1}-\delta_{i}
$$

we obtain from equations (8.27) for $i-1 \leqq n \leqq i+2$ the equations

$$
\begin{aligned}
& \hat{\alpha} d_{i-1} p_{i+1}+\hat{\beta} d_{i-1} q_{i+1}+\hat{\gamma} d_{i-1} r_{i+1}+\hat{\delta} d_{i-1} s_{i+1}=0 \\
& \hat{\alpha}\left(d_{i} p_{i+2}+c_{i} p_{i+1}\right)+\hat{\beta}\left(d_{i} q_{i+2}+c_{i} q_{i+1}\right)+\hat{\gamma}\left(d_{i} r_{i+2}+c_{i} r_{i+1}\right)
\end{aligned}
$$


$(8.30)$

$$
\begin{aligned}
&+\hat{\delta}\left(d_{i} s_{i+2}+c_{i} s_{i+1}\right)=1 \\
& \hat{\alpha}\left(c_{i} p_{i}\right.\left.+d_{i-1} p_{i-1}\right)+\widehat{\beta}\left(c_{i} q_{i}+d_{i-1} q_{i-1}\right)+\hat{\gamma}\left(c_{i} r_{i}+d_{i-1} r_{i-1}\right) \\
&+\hat{\delta}\left(c_{i} s_{i}+d_{i-1} s_{i-1}\right)=0 \\
& \hat{\alpha} d_{i} p_{i}+\hat{\beta} d_{i} q_{i}+\hat{\gamma} d_{i} r_{i}+\hat{\delta} d_{i} s_{i}=0
\end{aligned}
$$

where we assume the third and fourth equations of (8.30) to be also satisfied for $i=m-1, m$, and the first equation of (8.30) also for $i=0$. From (8.30) we obtain

$$
\begin{aligned}
& \hat{\alpha}=-\frac{1}{d_{i}}|q r s|_{i} /|\operatorname{pqrs}|_{i}, \widehat{\beta}=\frac{1}{d_{i}}|p r s|_{i} /|p q r s|_{\iota}, \\
& \hat{\gamma}=-\frac{1}{d_{i}}|p q s|_{i} /|p q r s|_{i}, \hat{\delta}=\frac{1}{d_{i}}|p q r|_{i} /|p q r s|_{i}
\end{aligned}
$$

where

(8.32) $|p q r|_{i}=\left|\begin{array}{lll}p_{i-1} & q_{i-1} & r_{i-1} \\ p_{i} & q_{i} & r_{i} \\ p_{i+1} & q_{i+1} & r_{i+1}\end{array}\right|,|p q r s|_{i}=\left|\begin{array}{llll}p_{i-1} & q_{i-1} & r_{i-1} & s_{i-1} \\ p_{i} & q_{i} & r_{i} & s_{i} \\ p_{i+1} & q_{i+1} & r_{i+1} & s_{i+1} \\ p_{i+2} & q_{i+2} & r_{i+2} & s_{i+2}\end{array}\right|(i \geqq-1)$.

From (8.2), (8.28) we obtain using (2.1)

$$
\gamma_{i}=\cdots=\gamma_{-2}=0, \delta_{i}=\cdots=\delta_{-2}=0
$$

and then from (8.28), (8.29), (8.31), (8.33) we obtain

$$
\begin{aligned}
& \gamma_{m+2}=\cdots=\gamma_{i+1}=\hat{\gamma}=-\left.\frac{1}{d_{i}}|p q s|_{i}|| p q r s\right|_{i} \\
& \delta_{m+2}=\cdots=\delta_{i+1}=\hat{\delta}=\frac{1}{d_{i}}|p q r|_{i} /|p q r s|_{i} .
\end{aligned}
$$

From (8.3), (8.28), (8.34) and using also the fourth equation of (8.30) for $i=m$ and (8.29) we obtain

$$
\begin{aligned}
& \alpha_{m+2}=\cdots=\alpha_{i+1}=\left[-|p q s|_{i}|q r|_{m}+|p q r|_{i}|q s|_{m}\right] / d_{i}|p q r s|_{i}|p q|_{m} \\
& \beta_{m+2}=\cdots=\beta_{i+1}=\left[|p q s|_{i}|p r|_{m}-|p q r|_{i}|p s|_{m}\right] / d_{i}|p q r s|_{i}|p q|_{m}
\end{aligned}
$$

where $|p q|_{m}$ etc. are defined in (2.2), and then from (8.29), (8.35), (8.31), (8.28) we obtain

$$
\begin{aligned}
\alpha_{i}= & \cdots=\alpha_{-2}=\left[-|p q s|_{i}|q r|_{m}+|p q r|_{i}|q s|_{m}\right. \\
& \left.+|q r s|_{i}|p q|_{m}\right] / d_{i}|p q r s|_{i}|p q|_{m} \\
\beta_{i}= & \cdots=\beta_{-2}=\left[|p q s|_{i}|p r|_{m}-|p q r|_{i}|p s|_{m}\right. \\
& \left.-|p r s|_{i}|p q|_{m}\right] / d_{i}|p q r s|_{i}|p q|_{m} .
\end{aligned}
$$

We now see from (8.9), (8.10) that 


$$
|p q r s|_{i}=d_{i-1}^{-1} d_{i}^{-1} D_{i}=d_{i-1}^{-1} d_{i}^{-1} d_{-2} d_{-1} \quad(i \geqq-1)
$$

and expanding the determinant and using (3.1), (3.7) that

$$
\begin{aligned}
& |p q r|_{i}=-d_{-2} q_{i} / d_{i-1},|p q s|_{i}=-\left(c_{-1} q_{i}-d_{-1} p_{i}\right) / d_{i-1}, \\
& |p r s|_{i}=-\left(c_{-1} r_{i}-d_{-2} s_{i}\right) / d_{i-1},|q r s|_{i}=-d_{-1} r_{i} / d_{i-1} \quad(i \geqq-1) .
\end{aligned}
$$

Using (8.37), (8.38) in (8.33), (8.34), (8.35), (8.36), substituting in (8.26) and making use of relation (8.5) of Lemma 8.1 and (8.12) we obtain Green's function $g_{r i}^{(m)}(\lambda)$ in the form (8.16).

We now verify that (8.15), (8.16) is a solution of (8.1), (8.2), (8.3) with the aid of Lemmas 8.1, 8.2, 8.3, 8.4 and then show that the solution is unique.

Set $y_{r}(\lambda)=g_{r n}^{(m)}(\lambda) f_{n}$ in equation (8.1) for $n$. Then the left side becomes, using the recurrence relations (1.1) satisfied by $p_{n}, q_{n}$,

$$
\begin{aligned}
\left\{d_{n} g_{n+2, n}^{(m)}\right. & \left.+c_{n} g_{n+1, n}^{(m)}+\left(b_{n}-\lambda a_{n}\right) g_{n, n}^{(m)}+c_{n-1} g_{n-1, n}^{(m)}+d_{n-2} g_{n-2, n}^{(m)}\right\} f_{n} \\
= & \left\{d_{n}\left(p_{n} \psi_{1 n+2}+q_{n} \psi_{2 n+2}\right)+c_{n}\left(p_{n} \psi_{1 n+1}+q_{n} \psi_{2 n+1}\right)\right. \\
& +\left(b_{n}-\lambda a_{n}\right)\left(p_{n} \psi_{1 n}+q_{n} \psi_{2 n}\right)+c_{n-1}\left(\psi_{1 n} p_{n-1}+\psi_{2 n} q_{n-1}\right) \\
& \left.+d_{n-2}\left(\psi_{1 n} p_{n-2}+\psi_{2 n} q_{n-2}\right)\right\} a_{n} f_{n}=\left\{d_{n}\left(p_{n} \psi_{1 n+2}+q_{n} \psi_{2 n+2}\right)\right. \\
& +c_{n}\left(p_{n} \psi_{1 n+1}+q_{n} \psi_{2 n+1}\right)-d_{n}\left(\psi_{1 n} p_{n+2}+\psi_{2 n} q_{n+2}\right) \\
& \left.-c_{n}\left(\psi_{1 n} p_{n+1}+\psi_{2 n} q_{n+1}\right)\right\} a_{n} f_{n}=\left\{\left[\psi_{1} \bar{p}\right]+\left[\psi_{2} \bar{q}\right]\right. \\
& \left.-d_{n-1}\left(\psi_{1 n+1} p_{n-1}-\psi_{1 n-1} p_{n+1}\right)-d_{n-1}\left(\psi_{2 n+1} q_{n-1}-\psi_{2 n-1} q_{n+1}\right)\right\} a_{n} f_{n} \\
= & (1+1-1) a_{n} f_{n}=a_{n} f_{n}
\end{aligned}
$$

using (8.14) of Lemma 8.4, (8.11) and relation (8.8) of Lemma 8.2.

Hence this term in the expression (8.15) for $y_{r}(\lambda)$ satisfies equation (8.1) for $n$.

Set $y_{r}(\lambda)=g_{r n-1}^{(m)}(\lambda) f_{n-1}$ in equation (8.1) for $n$. Then we obtain, using the fact that the $\psi_{i n}$ satisfy recurrence relations (1.1),

$$
\begin{aligned}
& \left\{d_{n} g_{n+2, n-1}^{(m)}+c_{n} g_{n+1, n-1}^{(m)}+\left(b_{n}-\lambda a_{n}\right) g_{n, n-1}^{(m)}+c_{n-1} g_{n-1 n-1}^{(m)}\right. \\
& \left.\quad+d_{n-2} g_{n-2, n-1}^{(m)}\right\} f_{n-1}=\left\{d_{n}\left(p_{n-1} \psi_{1 n+2}+q_{n-1} \psi_{2 n+2}\right)\right. \\
& \quad+c_{n}\left(p_{n-1} \psi_{1 n+1}+q_{n-1} \psi_{2 n+1}\right)+\left(b_{n}-\lambda a_{n}\right)\left(p_{n-1} \psi_{1 n}+q_{n-1} \psi_{2 n}\right) \\
& \quad+c_{n-1}\left(p_{n-1} \psi_{1 n-1}+q_{n-1} \psi_{2 n-1}\right)+d_{n-2}\left(\psi_{1 n-1} p_{n-2}+\psi_{2 n-1} q_{n-2}\right\} a_{n-1} f_{n-1} \\
& =d_{n-2}\left(\psi_{1 n-1} p_{n-2}+\psi_{2 n-1} q_{n-2}-\psi_{1 n-2} p_{n-1}-\psi_{2 n-2} q_{n-1}\right) d_{n-1} f_{n-1}=0
\end{aligned}
$$

using Lemma 8.4 and relation (8.5) of Lemma 8.1 for $h, k=0$.

Similarly we see that $y_{r}(\lambda)=g_{r n+1}^{(m)}(\lambda) f_{n+1}$ in the left side of equation (8.1) for $n$ gives 0 . Also if we set $y_{r}(\lambda)=g_{r s}^{(m)}(\lambda) f_{s}, s \leqq n-2$ or $s \geqq n+2$, in equation (8.1) for $n$, we obtain, using the fact that the $\psi_{i n}^{(m)}$ satisfy recurrence relations (1.1), that the left side of equation becomes 0 .

Hence we can state that $y_{r}(\lambda)$ as given by (8.15) satisfies the recurrence relations (8.1). 
Now $y_{r}(\lambda)$ given in (8.15) can easily be seen to satisfy initial conditions (8.2).

We now show that conditions (8.3) are satisfied.

Set $y_{r}(\lambda)=g_{r n}^{(m)}(\lambda) f_{n}$ in the relations (8.3). Then the left side of the first relation becomes for $n \leqq m-1$

$$
\begin{aligned}
& y_{m+1}+k\left[c_{m} y_{m}+d_{m-1} y_{m-1}\right]=\left\{g_{m+1, n}^{(m)}+k\left[c_{m} g_{m, n}^{(m)}+d_{m-1} g_{m-1, n}^{(m)}\right]\right\} f_{n} \\
&=\left\{p_{n} \psi_{1 m+1}+q_{n} \psi_{2 m+1}+k\left[c_{m} p_{n} \psi_{1 m}+c_{m} q_{n} \psi_{2 m}+d_{m-1} p_{n} \psi_{1 m-1}\right.\right. \\
&\left.\left.+d_{m-1} q_{n} \psi_{2 m-1}\right]\right\} a_{n} f_{n}=0
\end{aligned}
$$

since the $\psi_{i}^{(m)}(i=1,2)$ satisfy boundary conditions (8.3).

Similarly for $n \leqq m$ we have

$$
\begin{aligned}
y_{m+2}+h y_{m} & =\left\{g_{m+2, n}^{(m)}+h g_{m, n}^{(m)}\right\} a_{n} f_{n} \\
& =\left\{p_{n} \psi_{1 m+2}+q_{n} \psi_{2 m+2}+h p_{n} \psi_{1 m}+h q_{n} \psi_{2 m}\right\} a_{n} f_{n}=0 .
\end{aligned}
$$

For $n=m$ we have, using the fact that the $\psi_{i n}^{(m)}(i=1,2)$ satisfy boundary conditions (8.3),

$$
\begin{aligned}
y_{m+1}+ & k\left[c_{m} y_{m}+d_{m-1} y_{m-1}\right]=\left\{g_{m+1, m}^{(m)}+k\left[c_{m} g_{m, m}^{(m)}+d_{m-1} g_{m-1, m}^{(m)}\right]\right\} f_{m} \\
= & \left\{p_{m} \psi_{1 m+1}+q_{m} \psi_{2 m+1}+k\left[c_{m} p_{m} \psi_{1 m}+c_{m} q_{m} \psi_{2 m}+d_{m-1} p_{m-1} \psi_{1 m}\right.\right. \\
& \left.\left.+d_{m-1} q_{m-1} \psi_{2 m}\right]\right\} a_{m} f_{m}=k d_{m-1}\left\{\psi_{1 m} p_{m-1}+\psi_{2 m} q_{m-1}\right. \\
& \left.-\psi_{1 m-1} p_{m}-\psi_{2 m-1} q_{m}\right\} a_{m} f_{m}=0
\end{aligned}
$$

using relation (8.14) of Lemma (8.4) and (8.5) of Lemma 8.1 for $h=$ $k=0$.

Hence $y_{r}(\lambda)$ as given in formula (8.15) satisfies conditions (8.3).

The uniqueness of the solution follows from the fact that, since $\lambda$ is not an eigenvalue of the corresponding homogeneous boundary problem (1.1), (1.2), (1.3), the difference of two solutions of (8.1), (8.2), (8.3) must be the trivially zero solution.

To prove (8.17) we require the following lemma giving an identity analogous to Green's formula for differential equations:

LeMma 8.5. For two vectors $u=\left\{u_{n}\right\}, v=\left\{v_{n}\right\}(-2 \leqq n \leqq m+2)$ of complex numbers $u_{n}, v_{n}$ we have the formula

$$
\sum_{n=0}^{m}(P u)_{n} \bar{v}_{n}-u_{n}\left(\overline{p^{v}}\right)_{n}=[u v]_{m}-[u v]_{-1}
$$

where the form $[u v]_{n}$ is defined in (3.1) and the difference operator $P$ acting on a sequence $u=\left\{u_{n}\right\}(-2 \leqq n \leqq m+2)$ of complex numbers $u_{n}$ is defined by

$$
(P u)_{n}=d_{n} u_{n+2}+c_{n} u_{n+1}+b_{n} u_{n}+c_{n-1} u_{n-1}+d_{n-2} u_{n-2} \quad(0 \leqq n \leqq m)
$$

The proof of (8.39) is immediate from the form of (8.40). 
We now can obtain (8.17) of Theorem 8.1. We set in (8.39) $u=$ $v=y$ where $y$ is the solution of (8.1), (8.2), (8.3). Then from (8.2), (8.3) we see that $[y y]_{m}=[y y]_{-1}=0$ and from (8.1) $(P y)_{n}=\lambda a_{n} y+a_{n} f_{n}$. Then (8.39) may be written

$$
2 i \operatorname{Im} \lambda \sum_{n=0}^{m} a_{n}\left|y_{n}\right|^{2}=\sum_{n=0}^{m} a_{n}\left(\bar{f}_{n} y_{n}-f_{n} \bar{y}_{n}\right) \text {. }
$$

We deduce with the aid of the Schwarz inequality

$$
\begin{aligned}
2|\operatorname{Im} \lambda| \sum_{n=0}^{m} a_{n}\left|y_{n}\right|^{2} & =\left|\sum_{n=0}^{m} a_{n}\left(\bar{f}_{n} y_{n}-f_{n} \bar{y}_{n}\right)\right| \\
& \leqq 2\left(\sum_{n=0}^{m} a_{n}\left|f_{n}\right|^{2}\right)^{1 / 2}\left(\sum_{n=0}^{m} a_{n}\left|y_{n}\right|^{2}\right)^{1 / 2}
\end{aligned}
$$

and hence

$$
\|y\|_{m} \leqq\left.|\operatorname{Im} \lambda|^{-1}|| f\right|_{m}
$$

where $\|y\|_{m}$ is given by (8.18), and we have the result (8.17).

Now (8.19) of (i) follows from the form of $g_{r i}^{(m)}(\lambda)$ given in (8.16) with (8.12). (8.23), (8.24) and (8.25) of (v) follow from the form of the solution $y$ of (8.1), (8.2), (8.3) given by (8.15) and hence from the fact that $\left\{g_{r i}(\lambda)\right\}_{r=-2}^{m+2}$ satisfies (8.1) with $\left\{f_{n}\right\}_{0}^{m}=\left\{\delta_{n i}\right\}_{n=0}^{m}$, and (8.2), (8.3). Hence also (8.20) of (ii) follows from (8.17) using $\left\|\delta_{n i}\right\|_{m}=a_{i}^{1 / 2}$. From (8.20) we easily deduce (8.21) of (iii). (8.22) of (iv) follows from (8.20) using (8.19). This completes the proof of Theorem 8.1.

We obtain the following corollary:

COROLLARY 8.1. The solution of the inhomogeneous recurrence relations (8.1) satisfying the initial conditions

$$
y_{-2}=y_{-1}=y_{0}=y_{1}=0
$$

is given by $y=y_{n}(\lambda)(n \geqq-2)$ where

$$
y_{n}=\sum_{k=1}^{4}\left(\sum_{i=0}^{n-2} v_{k i} a_{i} f_{i}\right) \phi_{k n}
$$

where $\phi_{k}=\left\{\phi_{k n}(\lambda)\right\}_{n=-2}^{m+2}(1 \leqq k \leqq 4)$ are four linearly independent solutions of the homogeneous recurrence relations (1.1) and the vectors $v_{k}=\left\{v_{k i}(\lambda)\right\}_{i=-2}^{m+2}(1 \leqq k \leqq 4)$ are four linearly independent solutions forming the adjoint fundamental systems of solutions of (1.1) and are given by

$$
\begin{aligned}
& v_{1 i}=\left\{-\left[\phi_{3} \bar{\phi}_{4}\right] \phi_{2 i}+\left[\phi_{2} \bar{\phi}_{4}\right] \phi_{3 i}-\left[\phi_{2} \bar{\phi}_{3}\right] \phi_{4 i}\right\} / D_{-1} \\
& v_{2 i}=\left\{\left[\phi_{3} \bar{\phi}_{4}\right] \phi_{1 i}-\left[\phi_{1} \bar{\phi}_{4}\right] \phi_{3 i}+\left[\phi_{1} \bar{\phi}_{3}\right] \phi_{4 i}\right\} / D_{-1} \\
& v_{3 i}=\left\{-\left[\phi_{2} \bar{\phi}_{4}\right] \phi_{1 i}+\left[\phi_{1} \bar{\phi}_{4}\right] \phi_{2 i}-\left[\phi_{1} \bar{\phi}_{2}\right] \phi_{4 i}\right\} / D_{-1} \\
& v_{4 i}=\left\{\left[\phi_{2} \bar{\phi}_{3}\right] \phi_{1 i}-\left[\phi_{1} \bar{\phi}_{3}\right] \phi_{2 i}+\left[\phi_{1} \bar{\phi}_{2}\right] \phi_{3 i}\right\} / D_{-1} \quad(i \geqq-2)
\end{aligned}
$$


where $D_{-1}=d_{-2} d_{-1}\left|\phi_{1} \phi_{2} \phi_{3} \phi_{4}\right|_{-1}$, and $\left|\dot{\phi}_{1} \phi_{2} \phi_{3} \phi_{4}\right|_{-1}$ is defined analogously to (8.32) and the forms $\left[\phi_{k} \bar{\phi}_{j}\right]$ are defined in (3.6), (3.1).

Proof. We note that the solution of (8.1), (8.41) exists and is unique for all $\lambda$ since the corresponding homogeneous equations (1.1) with (8.41) can have only the trivial zero solution.

We solve the equations (8.1), (8.41) by the method of variation of parameters setting

$$
y_{n}(\lambda)=\alpha_{n} \dot{\phi}_{1 n}(\lambda)+\beta_{n} \phi_{2 n}(\lambda)+\gamma_{n} \phi_{3 n}(\lambda)+\delta_{n} \phi_{4 n}(\lambda) \quad(n \geqq-2) .
$$

Analogously to the demonstration of (8.15), (8.16) of Theorem 8.1 we obtain the solution $y$ in the form (8.42) with

$$
\begin{aligned}
& v_{1 i}=-\left|\dot{\phi}_{2} \dot{\phi}_{3} \phi_{4}\right|_{i} / d_{i}\left|\phi_{1} \phi_{2} \phi_{3} \phi_{4}\right|_{i} \\
& v_{2 i}=\left|\phi_{1} \phi_{3} \phi_{4}\right|_{i} / d_{i}\left|\phi_{1} \phi_{2} \phi_{3} \phi_{4}\right|_{i} \\
& v_{3 i}=-\left|\phi_{1} \dot{\phi}_{2} \phi_{4}\right|_{i} / d_{i}\left|\phi_{1} \dot{\phi}_{2} \phi_{3} \phi_{4}\right|_{i} \\
& v_{4 i}=\left|\phi_{1} \phi_{2} \phi_{3}\right|_{i} / d_{i}\left|\phi_{1} \phi_{2} \phi_{3} \phi_{4}\right|_{i}
\end{aligned}
$$

where $\left|\phi_{1} \phi_{2} \phi_{3}\right|_{i},\left|\phi_{1} \phi_{2} \phi_{3} \phi_{4}\right|_{i}$ are defined analogously to (8.32), and we make use of the easily verifiable fact that

$$
\sum_{k=1}^{4} v_{k n-1} \phi_{k n}=0 \quad(n \geqq-1)
$$

Using recurrence relations (1.1) we obtain analogously to (8.37), (8.38) that

$$
\left|\phi_{1} \dot{\phi}_{2} \dot{\phi}_{3}\right|_{i}=\frac{1}{d_{i-1}}\left\{\left[\phi_{2} \bar{\phi}_{3}\right] \dot{\phi}_{1 i}-\left[\phi_{1} \bar{\phi}_{3}\right] \phi_{2 i}+\left[\phi_{1} \bar{\phi}_{2}\right] \phi_{3 i}\right\} \quad(i \geqq-1)
$$

etc. and

$$
\left|\phi_{1} \phi_{2} \phi_{3} \phi_{4}\right|_{i}=d_{-2} d_{-1}\left|\phi_{1} \phi_{2} \phi_{3} \dot{\phi}_{4}\right|_{-1} / d_{i-1} d_{i} \quad(i \geqq-1)
$$

and hence obtain $v_{k}(1 \leqq k \leqq 4)$ as in (8.43).

We see from (8.43) that the $v_{k}(1 \leqq k \leqq 4)$ are solutions of (1.1).

From (8.47) or directly from (1.1) we deduce the following corollary:

COROLLARY 8.2. If for four solution vectors $\phi_{k}=\left\{\phi_{k n}\right\}_{n=-2}^{m+2}(1 \leqq k \leqq 4)$ of (1.1) we have $\left|\dot{\phi}_{1} \phi_{2} \phi_{3} \phi_{4}\right|_{i} \neq 0$ for some $i \geqq-1$ then we have $\left|\phi_{1} \phi_{2} \phi_{3} \phi_{4}\right|_{i} \neq 0$ for all $i \geqq-1$. A necessary and sufficient condition for the solution vectors of (1.1) $\phi_{k}(1 \leqq k \leqq 4)$ to be linearly independent is that $\left|\phi_{1} \phi_{2} \dot{\phi}_{3} \dot{\phi}_{4}\right|_{i} \neq 0$ for some $i \geqq-1$.

We now verify that the $v_{k}(1 \leqq k \leqq 4)$ are linearly independent by showing, as is sufficient in view of (1.1), that

$$
\sum_{k=1}^{4} \alpha_{k} v_{k i}=0 \quad(i=-1,0,1,2)
$$


where the $\alpha_{k}(1 \leqq k \leqq 4)$ are complex constants implies that $\alpha_{k}=0$ $(1 \leqq k \leqq 4)$. For we may write (8.48) in view of (8.45) in the form

$$
\left|\begin{array}{llll}
\phi_{1 i-1} & \phi_{2 i-1} & \phi_{3 i-1} & \phi_{4 i-1} \\
\phi_{1 i} & \phi_{2 i} & \phi_{3 i} & \phi_{4 i} \\
\phi_{1 i+1} & \phi_{2 i+1} & \phi_{3 i+1} & \phi_{4 i+1} \\
\alpha_{1} & \alpha_{2} & \alpha_{3} & \alpha_{4}
\end{array}\right|=0 \quad(-1 \leqq i \leqq 2)
$$

Using the recurrence relations (1.1) we deduce that

$$
\left|\begin{array}{lllll}
\phi_{1 i-1} & \phi_{2 i-1} & \phi_{3 i-1} & \phi_{4 i-1} & \gamma_{1} \\
\phi_{1 i} & \phi_{2 i} & \phi_{3 i} & \phi_{4 i} & \gamma_{2} \\
\phi_{1 i+1} & \phi_{2 i+1} & \phi_{3 i+1} & \phi_{4 i+1} & \gamma_{3} \\
\phi_{1 i+2} & \phi_{2 i+2} & \phi_{3 i+2} & \phi_{4 i+2} & \gamma_{4} \\
\alpha_{1} & \alpha_{2} & \alpha_{3} & \alpha_{4} & 0
\end{array}\right|=0 \quad(i=0)
$$

for all complex constants $\gamma_{k}(1 \leqq k \leqq 4)$.

Since by Corollary $8.2\left|\phi_{1} \phi_{2} \phi_{3} \phi_{4}\right|_{0} \neq 0$ since the $\phi_{k}(1 \leqq k \leqq 4)$ are linearly independent, we obtain for some set of complex constants $\beta_{k}(1 \leqq k \leqq 4)$ not all zero and independent of the $\gamma_{k}(1 \leqq k \leqq 4)$ that

$$
\left|\begin{array}{lllll}
\phi_{1 i-1} & \phi_{2 i-1} & \phi_{3 i-1} & \phi_{4 i-1} & \gamma_{1} \\
\phi_{1 i} & \phi_{2 i} & \phi_{3 i} & \phi_{4 i} & \gamma_{2} \\
\phi_{1 i+1} & \phi_{2 i+1} & \phi_{3 i+1} & \phi_{4 i+1} & \gamma_{3} \\
\phi_{1 i+2} & \phi_{2 i+2} & \phi_{3 i+2} & \phi_{4 i+2} & \gamma_{4} \\
0 & 0 & 0 & 0 & \sum_{k=1}^{4} \gamma_{k} \beta_{k}
\end{array}\right|=0 \quad(i=0)
$$

and hence, since $\left|\phi_{1} \phi_{2} \phi_{3} \phi_{4}\right|_{0} \neq 0$ by Corollary 8.2, that $\sum_{k=1}^{4} \gamma_{k} \beta_{k}=0$ for all $\gamma_{k}(1 \leqq k \leqq 4)$. Hence $\beta_{k}=0(1 \leqq k \leqq 4)$. This is a contradiction and hence $\alpha_{k}=0(1 \leqq k \leqq 4)$ and the $v_{k}(1 \leqq k \leqq 4)$ are linearly independent.

We now derive the solution of the boundary problem (8.1), (8.2), (8.3) in an alternative form involving a different expression for the Green's function.

We obtain the following theorem:

THEOREM 8.2. The solution of the inhomogeneous boundary problem (8.1), (8.2), (8.3), provided $\lambda$ is not an eigenvalue of the corresponding homogeneous boundary problem (1.1), (1.2), (1.3), is given by

$$
y_{n}(\lambda)=\sum_{i=0}^{m} g_{n i}^{(m)}(\lambda) f_{i} \quad(-2 \leqq n \leqq m+2)
$$

where the Green's function $g_{n i}^{(m)}(\lambda)$ is given by 


$$
g_{n i}^{(m)}(\lambda)=\int_{-\infty}^{\infty} Y_{n}(\mu) d \tau_{m, h, k}(\mu) Y_{i}^{*}(\mu) a_{i} /(\mu-\lambda)
$$

for $-2 \leqq n \leqq m+2,0 \leqq i \leqq m$, where $Y_{n}(\lambda)$ are the matrix polynomials defined in (7.1).

Proof. We use the dual orthogonality relations (7.8) and the recurrence relations (7.2) and (7.3), as well as (7.4), (7.9).

Assuming (8.49), (8.50) we obtain with the aid of the recurrence relations (7.2) that the left-hand side of equation (8.1) becomes

$$
\begin{aligned}
\sum_{i=0}^{m} \int_{-\infty}^{\infty}( & \mu-\lambda) a_{n} Y_{n}(\mu) d \tau_{m, h, k}(\mu) Y_{i}^{*}(\mu)(\mu-\lambda)^{-1} a_{i} f_{i} \\
& =\sum_{i=1}^{m} a_{n} \int_{-\infty}^{\infty} Y_{n}(\mu) d \tau_{m, h, k}(\mu) Y_{i}^{*}(\mu) a_{i} f_{i} \\
& =\sum_{i=1}^{m} a_{n} a_{i}^{-1} \delta_{n i} a_{i} f_{i} \\
& =a_{n} f_{n}
\end{aligned}
$$

using (7.8).

Hence the $y_{n}(\lambda)$ defined by (8.49), (8.50) satisfy the equations (8.1) and, as can readily be ascertained using (7.3), the initial conditions (8.2).

Also using the definition of $\tau_{m, h, k}(\lambda)$ given in (7.9) and relation (7.4) we see that $g_{n i}^{(m)}(\lambda)$ as given by (8.50) and consequently $y_{n}(\lambda)$ in (8.49) satisfy the boundary conditions (8.3).

In a subsequent paper (Billigheimer [4]), we consider the boundary problem of the recurrence relations (1.1), (1.2) in the singular case $m \rightarrow \infty$. Both forms of Green's function given in Theorem 8.1 and 8.2 are of use in these further investigations.

\section{REFERENCES}

1. F. V. Atkinson, Discrete and continuous boundary problems, Academic Press, New York, 1964.

2. J. H. Barrett, Disconjugacy of a self-adjoint differential equation of the fourth order, Pacific J. Math. 9 (1961), 25-37.

3. - Systems-disconjugacy of a fourth-order differential equation, Proc. Amer. Math. Soc. 12 (1961), 205-213.

4. C. E. Billigheimer, Singular boundary problems for a five-term recurrence relation, Amer. J. Math. 91 (1969), 1012-1048.

5. E. A. Coddington and N. Levinson, Theory of ordinary differential equations, McGraw Hill, New York, 1955.

6. W. N. Everitt, The Sturm-Liouville problem for fourth-order differential equations, Quart. J. Math. Oxford (2) 8 (1957), 146-160.

7. —, Fourth order singular differential equations, Math. Ann. 149 (1963), 320-340.

8. I. M. Glazman, On the theory of singular differential operators, Usp. Math. Nauk. 
N. S. (5, 6) 40 (1950), 102-135; Amer. Math. Soc. Trans. (1) 4 (1962), 331-372.

9. K. Kodaira, On ordinary differential equations of any even order and the corresponding eigenfunction expansions, Amer. J. Math. 72 (1950), 502-544.

10. W. Leighton and Z. Nehari, On the oscillation of solutions of self-adjoint linear differential equations of the fourth order, Trans. Amer. Math. Soc. 89 (1958), 325-377. 11. M. H. Stone, Linear transformations in Hilbert space, Amer. Math. Soc. Colloquium Publications, Vol. XV, New York, 1932.

12. H. Weyl, Über gewöhnliche Differentialgleichungen mit Singularitäten und die zugehörigen Entwicklungen willkürlicher Funktionen, Math. Ann. 68 (1910), 220-269.

Received September, 17, 1968, and in revised form April 1, 1970. This work was initiated in a part of a $\mathrm{Ph}$. D. thesis submitted to the University of Toronto in 1966. The author is indebted to Professor F. V. Atkinson who acted as supervisor for his helpful criticism and constant encouragement.

MCMASTER UNIVERSITY

Hamilton, ONTARIo, Canada 



\section{PACIFIC JOURNAL OF MATHEMATICS}

\section{EDITORS}

H. SAmelson

Stanford University

Stanford, California 94305

RICHARd PieRCe

University of Washington

Seattle, Washington 98105

\author{
J. DugundJI \\ Department of Mathematics \\ University of Southern California \\ Los Angeles, California 90007 \\ RICHARD ARENS \\ University of California \\ Los Angeles, California 90024
}

ASSOCIATE EDITORS
E. F. BECKENBACH
B. H. NeumanN
F. WOLF
K. YosHIDA

\section{SUPPORTING INSTITUTIONS}

UNIVERSITY OF BRITISH COLUMBIA

CALIFORNIA INSTITUTE OF TECHNOLOGY

UNIVERSITY OF CALIFORNIA

MONTANA STATE UNIVERSITY

UNIVERSITY OF NEVADA

NEW MEXICO STATE UNIVERSITY

OREGON STATE UNIVERSITY

UNIVERSITY OF OREGON

OSAKA UNIVERSITY

UNIVERSITY OF SOUTHERN CALIFORNIA
STANFORD UNIVERSITY

UNIVERSITY OF TOKYO

UNIVERSITY OF UTAH

WASHINGTON STATE UNIVERSITY

UNIVERSITY OF WASHINGTON

AMERICAN MATHEMATICAL SOCIETY CHEVRON RESEARCH CORPORATION TRW SYSTEMS

NAVAL WEAPONS CENTER 


\section{Pacific Journal of Mathematics}

\section{Vol. 35, No. $1 \quad$ September, 1970}

B. D. Arendt and C. J. Stuth, On the structure of commutative periodic semigroups ..................................... 1

B. D. Arendt and C. J. Stuth, On partial homomorphisms of semigroups .... 7

Leonard Asimow, Extensions of continuous affine functions ............ 11

Claude Elias Billigheimer, Regular boundary problems for a five-term recurrence relation ................................... 23

Edwin Ogilvie Buchman and F. A. Valentine, A characterization of the

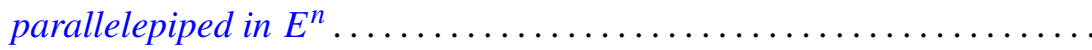
53

Victor P. Camillo, A note on commutative injective rings ............ 59

Larry Jean Cummings, Decomposable symmetric tensors ............. 65

J. E. H. Elliott, On matrices with a restricted number of diagonal values ... 79

Garth Ian Gaudry, Bad behavior and inclusion results for multipliers of type

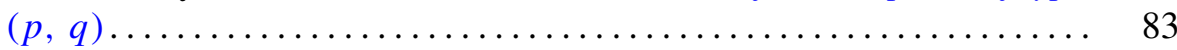

Frances F. Gulick, Derivations and actions .................. 95

Langdon Frank Harris, On subgroups of prime power index . . . . . . . . 117

Jutta Hausen, The hypo residuum of the automorphism group of an abelian

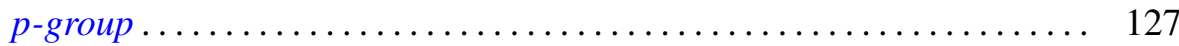

R. Hrycay, Noncontinuous multifuctions . ................... 141

A. Jeanne LaDuke, On a certain generalization of $p$ spaces ............ 155

Marion-Josephine Lim, Rank preservers of skew-symmetric matrices...... 169

John Hathway Lindsey, II, On a six dimensional projective representation of the Hall-Janko group ................................... 175

Roger McCann, Transversally perturbed planar dynamical systems . . . . . 187

Theodore Windle Palmer, Real $C^{*}$-algebras ................... 195

Don David Porter, Symplectic bordism, Stiefel-Whitney numbers, and a

Novikov resolution ....................................... 205

Tilak Raj Prabhakar, On a set of polynomials suggested by Laguerre

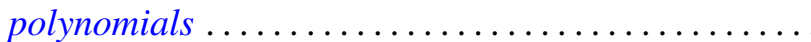

B. L. S. Prakasa Rao, Infinitely divisible characteristic functionals on locally convex topological vector spaces...................

John Robert Reay, Caratheodory theorems in convex product structures ...

Allan M. Sinclair, Eigenvalues in the boundary of the numerical range. .

David R. Stone, Torsion-free and divisible modules over matrix rings ....

William Jennings Wickless, A characterization of the nil radical of a

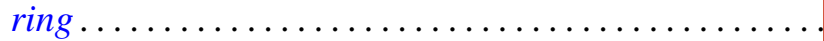

